\title{
The field guide to 3D printing in microscopy
}

\author{
Mario Del Rosario ${ }^{1, *}$, Hannah S. Heil ${ }^{1, *}$, Afonso Mendes ${ }^{1, *}$, Vittorio Saggiomo ${ }^{2}$, and Ricardo Henriques ${ }^{1,3 凶}$ \\ ${ }^{1}$ Optical Cell Biology, Instituto Gulbenkian de Ciência, Oeiras, Portugal \\ ${ }^{2}$ Laboratory of BioNanoTechnology, Wageningen University and Research, Wageningen, The Netherlands \\ ${ }^{3}$ Quantitative Imaging and Nanobiophysics, MRC Laboratory for Molecular Cell Biology, University College London, London, United Kingdom \\ These authors have contributed equally
}

\begin{abstract}
The maker movement has reached the optics labs, empowering researchers to actively create and modify microscope designs and imaging accessories. 3D printing has especially had a disruptive impact on the field, as it entails an accessible new approach in fabrication technologies, namely additive manufacturing, making prototyping in the lab available at low cost. Examples of this trend are taking advantage of the easy availability of 3D printing technology. For example, inexpensive microscopes for education have been designed, such as the FlyPi (1). Also, the highly complex robotic microscope OpenFlexure (2) represents a clear desire for the democratisation of this technology. 3D printing facilitates new and powerful approaches to science and promotes collaboration between researchers, as 3D designs are easily shared. This holds the unique possibility to extend the open-access concept from knowledge to technology, allowing researchers from everywhere to use and extend model structures. Here we present a review of additive manufacturing applications in microscopy, guiding the user through this new and exciting technology and providing a starting point to anyone willing to employ this versatile and powerful new tool.
\end{abstract}

3d printing | microscopy | open-source | optics | super-resolution Correspondence: rjhenriques@igc.gulbenkian.pt

\section{Introduction}

From the first microscopes invented in the late 16th century to the most recent iterations that can resolve targets beyond the diffraction limit of light, microscopy-based approaches represent a critical tool to study biological phenomena (3). Recent years have seen a sharp increase in bespoke microscopes, achieving excellent results where commercial solutions were ineffective, for example the the Warwick Open Source Microscope (WOSM) (https://wosmic.org) and the OMX Microscope (4). However, researchers are often limited to commercial parts, as fabrication methods to reliably manufacture microscope components are expensive and slow. This limitation hampers the diversity of new designs, ultimately restricting the innovation of new technology.

The approach of 3D printing holds great potential in this regard as it has had a disruptive impact on the field of manufacturing. This impact is based on its rapid prototyping approach to the creation of physical objects, called additive manufacturing. Additive manufacturing has seen a massive rise in popularity in recent years, from its start as an industrial prototyping tool to the current domestic use. It is employed across many different settings, from the user printing miniature figurines using common polymers in a small domestic printer (5) to entire houses using concrete in huge industrial machines (6). In 3D printing, a structure is built bottom-up in an

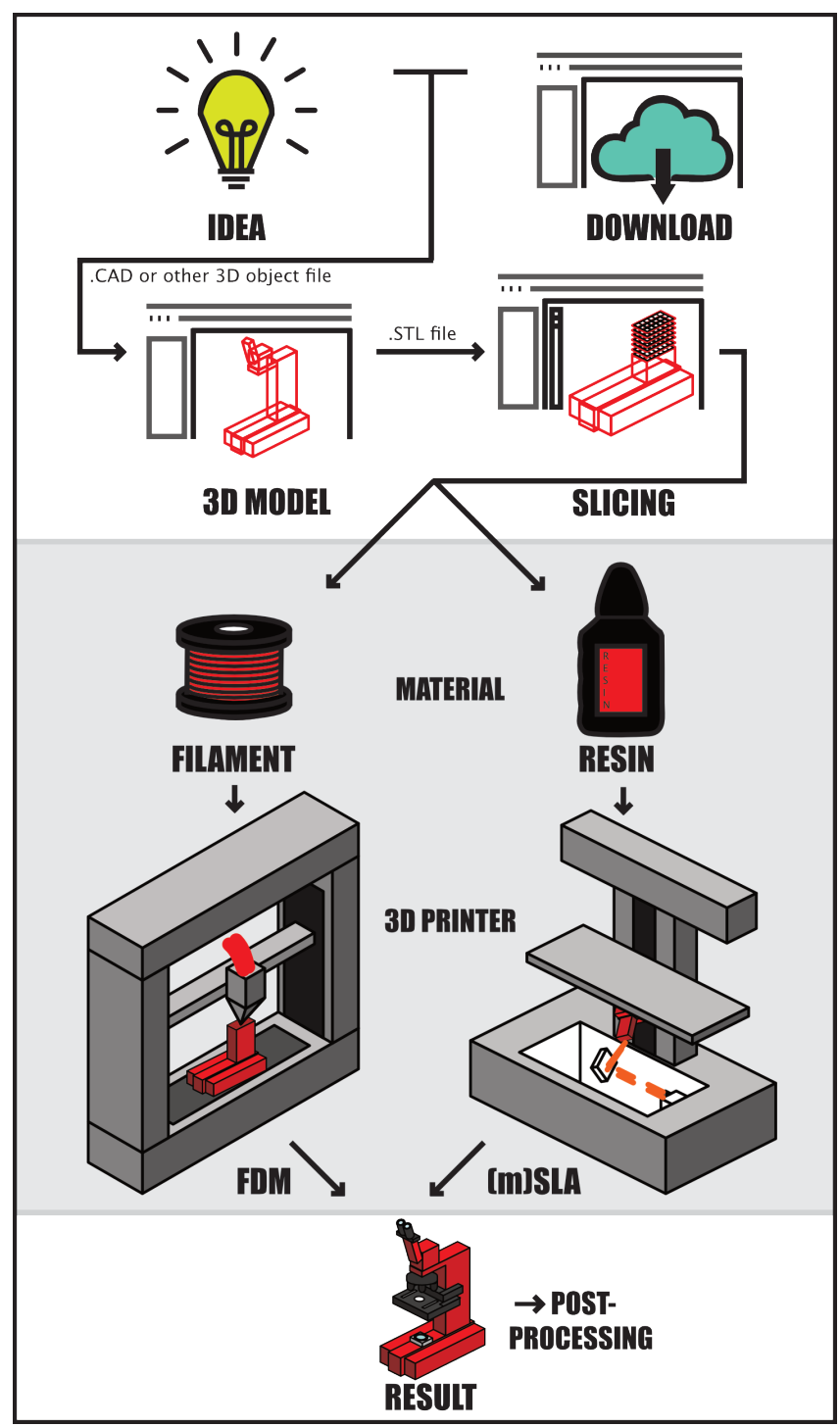

Fig. 1. $3 D$ printing process: from concept to reality. $3 D$ printing starts with an idea or a necessity to produce a specific object. A digital 3D model can be made "from scratch" or downloaded from an online database. The file encoding the 3D model needs to be processed in a slicing software, which divides the model into layers and creates instructions that the 3D printer can interpret. Depending on the desired results, different 3D printing technologies can be employed, which use different base materials (e.g. SLA uses resins and produces better details; FDM uses polymer filaments and produces high mechanical resistant products). Once the product is printed it might require further processing to achieve a high-quality finish (e.g. smooth surfaces). 
additive manner by depositing sub-mm layers of material. In contrast, conventional manufacturing processes usually rely on manual labour and automated processes such as casting, forming, and machining by either subtracting material from a larger starting piece or using moulds to shape an object (7). The additive manufacturing approach pursued in 3D printing uses simplified one-step manufacturing processes, reducing material waste, which in subtractive fabrication methods such as milling can be as high as $90 \%$ (8).

In scientific research, additive manufacturing is rapidly becoming a critical tool allowing the rapid development of new designs and the prototyping of machine components with an unprecedented speed. These components can be assembled to create complex machinery such as microscopes (2). Besides the advantage of rapid manufacturing, 3D printing facilitates novel and powerful approaches to solving problems in science, as it provides ample room for creativity. Additionally, $3 \mathrm{D}$ printing communities are well established, promoting collaborations between researchers and even the general public, as 3D designs are easily shared. 3D printing holds the unique possibility to extend the open-access concept from knowledge to technology, allowing researchers and people with a keen interest in science to use, iterate designs and adapt existing ones for different projects with a high degree of customisation.

\section{What is 3D printing?}

3D printing is a process that creates a physical object by adding layers of material to recreate a 3D digital object. Fig. 1 shows the typical framework used in 3D printing. This technology involves three main steps: the digital design of a $3 \mathrm{D}$ object, the computation of the printing instructions required by the printer and, finally, the fabrication of said object by adding patterned layers of new material. Depending on the application, vastly different 3D printing technologies that use different materials can be employed. This review focuses on two of the most popular methods: Fused Deposition Modelling (FDM) and Stereolithography (SLA). The printing process itself is as simple as melting plastic filaments in an extruder and using them to form layers that create mechanically strong objects (as done in FDM), or using light to selectively polymerise resins step by step to obtain highly intricate and complex forms (as done in SLA) or masked SLA (mSLA) (9).

Additive manufacturing starts with a 3D model designed from scratch or downloaded from the internet (Fig. 1). This is an exciting aspect of the technology, as files can be easily shared online, greatly increasing accessibility. The files are created and visualised in commercial or open-access 3D software (see also "Box 1. Software tools"). Once the 3D model is ready, it needs to be converted into a ".STL" file, which stands for Standard Tessellation Language. This is usually done by the CAD-type software itself but requires additional steps if using non-CAD based software. Once the .STL file is obtained, the printing pattern or path needs to be converted into a set of instructions that a 3D printer can interpret (i.e., for FDM, a "g-code"). This step is performed by a "slicer" software, which divides the object in layers and calculates the path that the printer needs to travel to produce them. The "slicing" software can also be commercial or open-access (see also "Box 1. Software tools" below).

Once a g-code file is created, the next step is 3D printing. The selection of materials and 3D printing technology will depend on various factors including cost, speed, material characteristics and needs. This review's scope is limited to commercial and common 3D printers, which use filaments and liquid resins to print with FDM and (m)SLA, respectively. Moreover, other printing technologies use different materials, including metals, ceramics, concrete, and even food (10). Depending on the machine used and the printing job, the printing process can be lengthy, taking over 1 day in some instances to complete. Once the object is printed, it can be subjected to post-processing steps.

\section{Box 1. Software tools: for 3D design:}

\section{D design software tools}

TinkerCAD: free, creation and rendering of 3D models, tutorials and teaching resources, interactive modeler and script based, very accessible even for unexperienced users, browser based (www.tinkercad.com)

OpenSCAD: Free, creation and rendering of 3D models, only script based. It might not be that intuitive to start with, but the short and comprehensible list of commands summarized on the cheatsheet is a great help. (www.openscad.org)

FreeCAD: free, creation and rendering, modeler based (www.freecadweb.org)

Blender: free, 3D model creation, rendering and animation

Fusion360: commercial software tool with free non-commercial subscription for one year (www.autodesk.com/products/fusion-360)

Rhino3D: commercial, free-form 3D modeling, creation, rendering and animation, handels complex models and point clouds (https://www.rhino3d.com) 3DS Max: commercial, 3D model creation, rendering and animation (www.autodesk.com/products/ 3ds-max)

SolidWorks: commercial, 3D model creation and rendering, includes motion and stress analysis tools. (www.solidworks.com)

\section{Free software tools for G-code creation}

- Slic3r: FDM (www.slic3r.org)

- PrusaSlicer: FDM (www.prusa3d.com)

- ideaMaker: FDM (www.ideamaker.io)

- Ultimaker Cura: FDM (https://ultimaker.com)

- PreForm: SLA (https://formlabs.com)

- chitubox: mSLA (https://www.chitubox.com) 


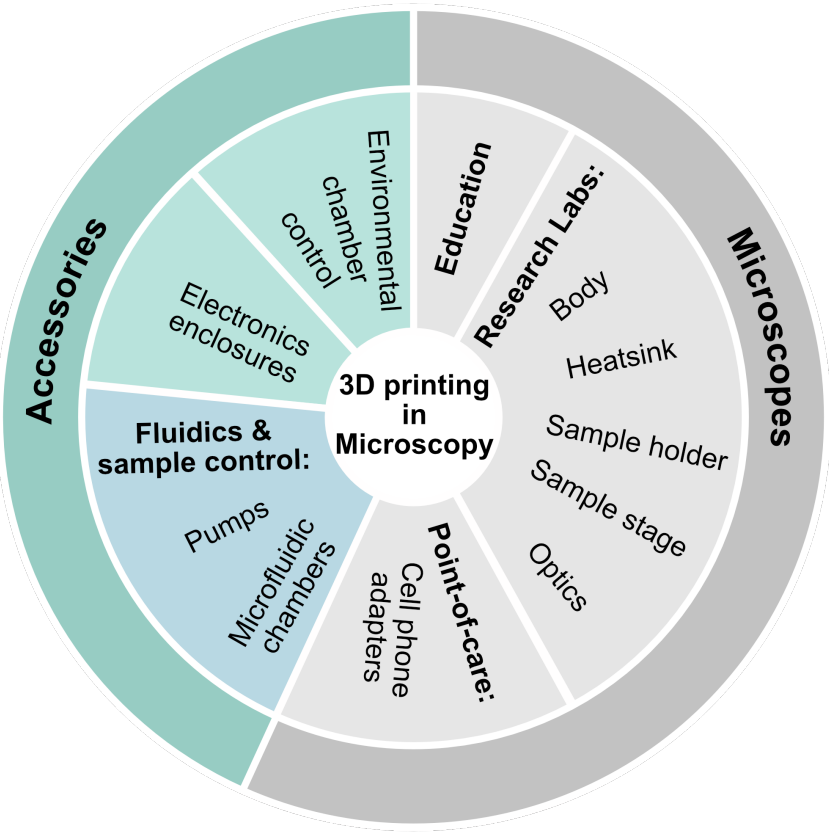

Fig. 2. Mindmap showing printed parts for microscopy applications. Additive manufacturing is currently used in many open source projects in microscope modular designs. With the exception of a few parts, it is currently possible to print whole microscope bodies at relatively low prices. Additionally, 3D printing is used for sample control such as microfluidics systems and sample holders.

For example, including support structures in the model is often required to print difficult areas (e.g., bridges and overhangs); these need to be removed manually afterwards, or dissolved in water if water-soluble filament is used (e.g. polyvinyl alcohol). Moreover, some models are printed in pieces that need to be assembled in an extra step. Finally, high-level surface finishes can be achieved by sanding, welding with organic solvents, painting, and polishing.

\section{D printing in microscopy}

With regard to the field of microscopy, 3D printing technologies are creating an impact in two critical aspects: accessibility and rapid prototyping of customised equipment. Along the lines of "Seeing is believing", microscopy plays a crucial role in studying biological processes by providing information far beyond the details perceived by the naked eye, down to the level of molecular organisation. From the very early days where Antonie van Leeuwenhoek observed protists and bacteria for the first time (11), to the modern super-resolution solutions capable of resolving images below the light diffraction limit ( $300 \mathrm{~nm})$, microscopy is the backbone of biology research (12).

Microscopy technology today is currently still limited in its application, often requiring high investment and specialised training. This puts researchers with limited funds at a disadvantage, granting access to sophisticated imaging approaches only to researchers from wealthy countries and facilities. This has given rise to a new movement in microfabrication and microscopy to create open access and inexpensive technology that can be accessible to a wider audience. In recent years, 3D printing-based solutions drastically increased the capability to design and fabricate scientific instruments, reducing fabrication time, cost, and structural limitations. More importantly, low-cost and highly sophisticated research tools are now accessible to a wider range of researchers, allowing a worldwide audience to benefit from better technology without relying on commercial solutions. 3D printing also facilitates iterative design-based approaches that would be difficult, if not impossible otherwise. Additionally, these benefits are available to the general public as well, as commercial 3D printers are now more accessible than ever. This aspect is particularly important because it enables "citizen scientists", empowering existing research avenues by increasing research output via crowd sourcing, which benefits society.

Many examples of this trend are taking advantage of the easy accessibility of 3D printing technology (see also Movie S1). For example, inexpensive microscopes for education are being produced, such as the FlyPi (1). Also, the highly complex robotic microscope OpenFlexure (3) represents a clear desire to democratise this technology. On the other hand, additive manufacturing is rapidly becoming the tool of choice in microscopy technology development, as 3D printing technology such as FDM allows rapid prototyping with an unprecedented level of freedom. This freedom in design paired with the unmatched speed in the generation of prototype components creates a perfect combination for innovating new cutting-edge technology where highly complex components can be fabricated and iterated upon with relative ease (Fig. 2). These characteristics enable systems such as miCube (13), the UC2 (14), and the the Warwick Open Source Microscope (WOSM) (https://wosmic.org), where further customisation is possible due to their modular design. The microscope parts that can be built vary with the technology and materials available. In the literature, whole microscope bodies have been printed, including the base $(13,15)$, the body $(13,15,16)$, holders for the filters $(15,17)$, objectives (18), coverslips (14), pinholes $(14,16)$ and heat sinks (18). Additionally, microscope chambers (19) and controller mounts (20) have also been implemented, allowing a high degree of customisation to researchers that adopt this technology.

3D printing is also used to print tools that allow for more complex microscopy solutions. Sample manipulation is an important aspect of this process that includes sample holders, sample surveying, and microfluidics systems (see also Movie S2). Sample holders are in direct contact with the sample, requiring biocompatible materials that are readily available or made biocompatible by post-processing. Examples include cell grid holders (21) and incubation chambers (22). Sample surveying using Atomic Force Microscopy cantilevers has also been 3D printed (23). Microfluidics can be used to control the movement of small liquid and particle volumes in a network of interconnected microchannels. Microfluidics is useful to dispense, mix, separate, and detect different reagents into a sample, allowing a high degree of manipulation using a system of automatic miniature pumps and Lab-on-Chip devices. Additionally, pump systems have been fully automatised to allow sample manipula- 
tion for downstream microscopy applications such as fixation and immunofluorescence $(24,25)$.

\section{D printed microscopy projects}

Microscopes are usually highly intricate machines composed of a multitude of parts with varying degrees of complexity. These parts have been traditionally built using standard micro- and macro- fabrication methods that are expensive and, in some cases, restrictive. The implementation of 3D printing approaches in the rapid fabrication and prototyping of optomechanical components allows the creation of new microscopy approaches, particularly when the designs incorporate methods to sense, process, and act automatically via computer-based controllers such as Arduino or Raspberry Pi. Using additive manufacturing to produce specific microscope components is highly beneficial, allowing fine-tuning to suit specific design requirements. It has been reported that $3 \mathrm{D}$ printing can reduce a microscope parts' price between $50 \%$ and $90 \%$, depending on the component (26). The performance of the 3D printed parts (kinematic mounts, translation stages, and integrating spheres) were directly compared to commercial counterparts to assess their precision performance. One of the tradeoffs that has to be accepted is the printed part's limited physical integrity, which can be compared to low-end commercial alternatives (26). Nevertheless, smartly designed and highly tailored components such as a monolithic 3D printed flexure translation stage have been realized with this approach. This stage was capable of submicron-scale motion with remarkably low drift and minimal post-processing (27). Micromanipulators and probe position systems have also been 3D printed and tested using flexible materials with substantial price reductions when compared to commercial options (28). Even tunable objectives have even been realized by aligning customised poly (methyl methacrylate) (PMMA) singlets and a miniaturized electrowetted lense for electronic focusing within a 3D-printed housing (29). While the fabrication of optically active elements usually requires nanoscale precision far beyond the capability of the 3D-printing techniques featured in this review, the implementation of near-refractive index matched media allows to realize a phasemask based on a 3D-printed mould with micrometer topography (30). Beyond this, low cost, compact and high-performance illumination systems have been realized employing 3D printed components, such as the NicoLase project. NicoLase is an open-source diode laser combiner, fiber launch, and illumination sequence controller for fluorescent microscopy and super-resolution applications that successfully competes with the performance of commercial systems at half of the costs (20).

The increase in mobile phone usage backed by powerful cameras and increasing computational output has given rise to the field of mobile microscopy. Although not as powerful as high-end dedicated cameras, it is still possible to use cellphone cameras to capture microscopy data after adapting hardware and software for this purpose. Mobile smartphones have achieved enough computational power that both acquisition and processing is now possible in the same device (31).
This practice can be particularly useful in fieldwork where conditions are not ideal for laboratory equipment. Projects such as cellSTORM have shown that it is possible to achieve SMLM using consumer mobile phones and achieve optical resolutions higher than $80 \mathrm{~nm}$. The project also benefits from using a trained image-to-image generative adversarial network (GAN) to reconstruct video sequences under suboptimal conditions, improving signal-to-noise ratio by compensating noise and compression artefacts (31). As the high performance scientific camera is usually one of the most expensive components of a microscope, exchanging it for a mobile phone camera allows to significantly reduce the overall costs of the system, even more so by integrating this approach into a 3D-printed microscope. This approach has been pursued for the FPscope project, capable of high-resolution imaging using variably illuminated, low-resolution intensity images in Fourier space, called Fourier ptychographic microscopy. Here, the mobile phone lens is used in a reversed manner where the mobile phone lens projects the magnified image to the detector (32). The $\mu$ SmartScope is another example of a $3 \mathrm{D}$ printing adaptor that can be fitted into a wide range of mobile smartphones. In addition, the motorised stage is fully automated and controllable by the smartphone and is capable of autonomous image acquisition (33).

Especially for particular tasks where the limited camera performance and computation power is sufficient, smartphones are used for microscopy applications in enabling point-ofcare and field diagnostics. These kinds of applications are for example DNA fluorescence spectroscopy for the readout of fluorescence-based biological assays to detect specific nucleic acid sequences reached the point of detecting single-base mutations (34). Other DNA-based applications include a surface-heated droplet PCR system to detect Escherichia coli that employs wire-guided droplet manipulation to guide a droplet over three different heating chambers. Following product amplification, end-point detection is achieved using the smartphone-based fluorescence microscope (35). Automated cell identification has also been done using a 3D printed shearing digital holographic microscope for fieldwork. It uses a common path shearing interferometer for automatic cell identification via a CMOS or a mobile phone camera and the addition of laser illumination and a commercial objective (36). Another holographic lens-less smartphone-based microscope has been designed for holographic micro-object imaging of a variety of samples using a CMOS camera chip and controlled by a Raspberry Pi (16). Further examples of smartphone-based projects differentiate between white blood cells with acridine orange staining using a miniature achromatic microscope (37), identify pathogenic bacteria using a DNA-based FISH assay (38), and image and identify malaria parasites with a ball lens objective capable of high-resolution bright field imaging of Plasmodium parasites in blood smears for field diagnostics (39).

Perhaps the most interesting application of 3D printing in microscopy is the generation of fully functioning microscopes with few non-3D printed components. 3D printed microscopes pose an exciting concept that combines iterative and 


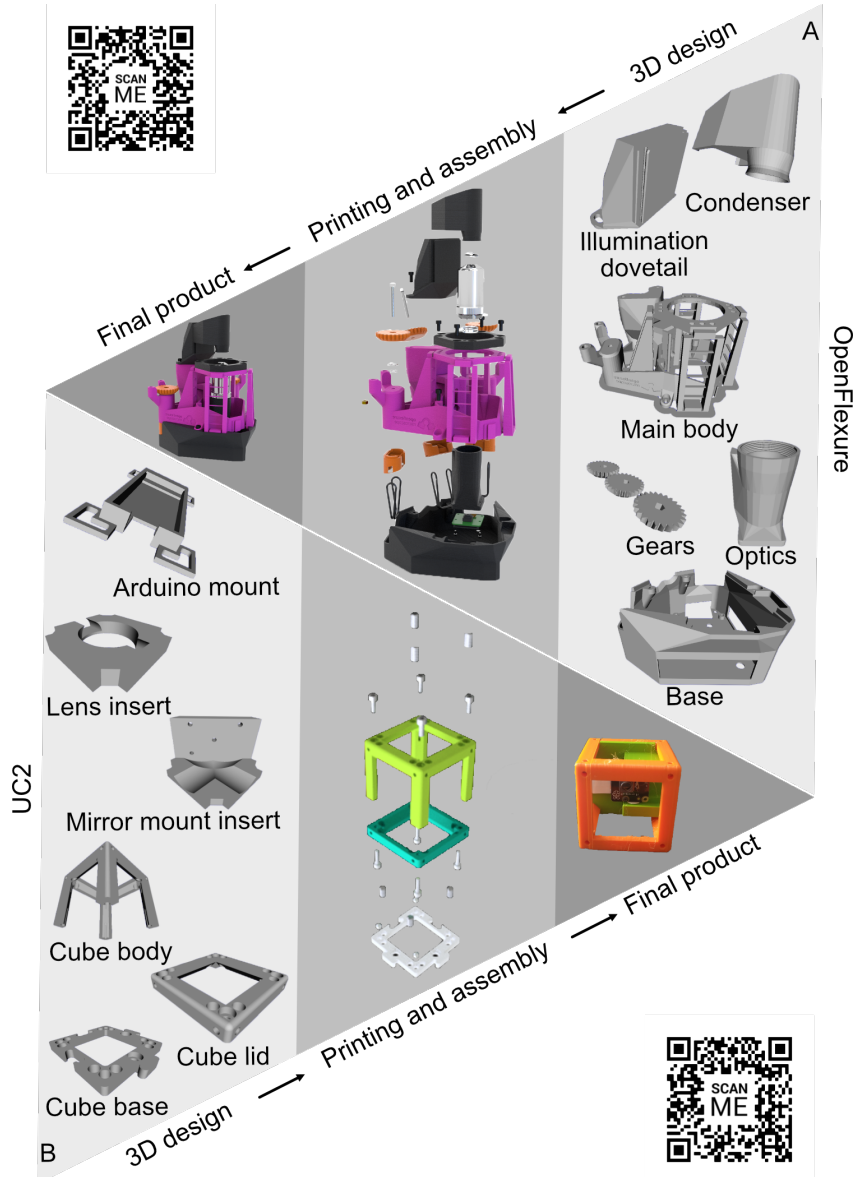

Fig. 3. 3D printed microscope parts. Microscope individual parts can be 3D printed and following post-processing, can be assembled to create a fully working microscope. (a) The OpenFlexure Microscope is an example of an almost fully 3Dprintable microscope (3). (b) The UC2 project (40) is based on cubic structures able to integrate inserts to allow microscopy techniques such as SIM. Certain parts can not be 3D printed and are sourced independently (e.g. lenses and electronics).

collaborative design, resulting in highly customisable equipment that would be very expensive to obtain using other fabrication methods. Thus, beyond the iterative design aspect, many 3D printed microscopes are open-source projects that present a unique opportunity to provide accessibility to novel ground-breaking designs to a wide range of researchers bypassing geographical and economic limitations.

Fully functioning 3D printed microscopes often present a modular design, allowing to add or remove components according to the needs of a particular experiment. For example, the $\mu$ Cube project uses cubes to create a framework for a 3D printable microscope using the parametric design of modular mounts. Additionally, it facilitates the alteration of the original design, allowing to generate new concepts (41).

One of the most prominent examples of open-source microscopy projects is the OpenFlexure microscope (Fig. 3a). OpenFlexure is a fully automated laboratory-grade microscope capable of using motorised sample positioning and focus control (3). Additionally, it is highly customisable, allowing trans- and epi- illumination, polarization contrast and epifluorescence imaging. It also uses high-end objectives and employs an 8MP CMOS sensor Raspberry Pi camera (V2) that was calibrated to use custom optics (42). The OpenFlex- ure microscope is controlled using the OpenFlexure software stack that is both cross-platform and language-independent. Control is split between a client and a server application that interface with each other using a web API with the W3C Web of Things standard. This characteristic provides a modern interface, multi-language support, minimizes code duplication, allows multiple microscopes to be controlled by a single computer, and integrates research experiments, users, and equipment (43). The OpenFlexure project was co-developed between the University of Bath and the Tanzanian company STICLab. Co-development of a project such as this shows the potential of open-source $3 \mathrm{D}$ printing projects where geographical boundaries no longer limit scientists from lowincome countries to access better resources, improving their research freedom (2). The OpenFlexure project has also been combined with SRRF (44) for super-resolution applications (45).

Another example of a 3D printed microscope with superresolution capacity is the Chea(i)p, a self-contained superresolution microscope that uses a commercial objective and a mobile phone, and costs less than $€ 800$. In this case, the mobile phone does the acquisition, processing, hardware control, and photonic-chip illumination. Impressively, it can reach resolutions of $100 \mathrm{~nm}$ with SMLM and live superresolution with SRRF (46). Furthermore, the waveguidePAINT system allows a highly uniform $100 \times 2000 \mu^{2}$ area evanescent field for TIRF illumination. The system was developed as a stable, low-cost microscope with a 3Dprintable chip holder to facilitate alignment and imaging. The waveguide-PAINT is capable of imaging multiple whole cells or whole origami structures such as microtubules in COS-7 cells in a single field of view (47).

3D printing has also been adapted to Selective Plane Illumination Microscopy (SPIM). SPIM, also known as light-sheet microscopy, is extremely useful for volumetric imaging of large samples as optical sectioning is achieved by employing a sheet of light as illumination. The OpenSPIM project is an open-access platform that allows new users to build a basic SPIM microscope step-by-step (18). Beyond applications in education and scientific outreach (48), the system can be upgraded and adapted for specific requirements and budgets. Although considered a challenging endeavour, the OpenSPIM was adapted and used to image the organism Maritigrella crozieri using two-colour laser illumination to simultaneously detect two probes at the same time (49). Another SPIM 3D printed system is the Flamingo, which offers the possibility of a highly customisable microscope that is adaptable to individual needs and portable [https://involv3d.org]. This allows to provide access to SPIM systems to researches all over the world as travelling shareable instruments.

The CellScope project uses a programmable domed LED array, enabling simultaneous multi-contrast imaging in brightfield, darkfield, and phase imaging modes. It works by scanning through illumination angles that capture light field datasets. These datasets recover 3D intensity and phase images without hardware alterations. It can also refocus digitally to achieve either 3D imaging or software-based correc- 
tion, allowing to bypass the necessity of precise mechanical focusing during acquisition (50).

One of the goals to achieve wide accessibility to microscopy technology is to substantially reduce the price by either 3D printing or procuring commonly used laboratory parts. Here great examples are the OPN Scope that aims to lower the costs for fluorescence microscopy (51) and the FlyPi, which uses a 3D-printed mainframe, a Raspberry Pi computer, a high-definition camera system and Arduino-based optical and thermal control circuits for $€ 200$ or less. The system was tested in experiments involving behavioural tracking in C. elegans as well as Optogenetics and Thermogenetics in Drosophila and C. elegans (1). The miCube open-microscopy framework is another system of this family. It has been used to visualise dCas9 in vivo target search, as it is capable of single-molecule microscopy with high spatiotemporal resolution (13). The Microscopi project aims to democratise microscopy with their portable, low cost, 3D printed and selfbuilt systems capable of multimodal imaging (bright field, dark field, pseudo-phase and fluorescent microscopy). It uses an automated XYZt imaging system controlled by a tablet or smartphone using a simple GUI (52).

Lastly, the UC2 (You. See. Too.) project is a low-cost, highly versatile and customisable 3D printed microscope that uses a modular design toolbox. The system is fully accessible online and uses many common off-the-shelf optics and electronic components that are fitted in 3D printed cubes (Fig. 3b). It has been used to acquire macrophage cell differentiation data, as well as apoptosis and proliferation enclosed in an integrated incubator in one of the modules and by minimizing axial drift with an automated focusing system (14).

\section{Sample Manipulation, microscopy and 3D printing}

3D printing applications for sample manipulation are a highly versatile and powerful tool. Especially the field of microfluidics is a highly interesting target for applying additive manufacturing, allowing to downscale biochemical applications to the point of creating portable and nano-scale versions of a testing laboratory. While originally the field was based on moulding and replica molding fabrication, today 3D printing allows the creation of more complex geometries and designs. With this approach, the production of moving parts is possible, including miniaturised pumps and valves that allow accurate fluidics control, as well as sensors that allow the detection of microchanges in the environment (Fig. 4). Additionally, the variety of 3D printing materials available allows researchers to customise the parts to suit specific needs (53). Functional parts such as valves are divided into manual and pneumatic, with the former requiring manual control and the latter requiring an energy source. Several examples of valves exist in the literature, ranging in complexity. Simple manual valves consist of an enclosed valve that allows flow when the inside and outside channels are aligned in the opened position $(53,54)$. Other more complex valves such as pneumatic ones, can be printed in arrays to mimic circuits (55) or quake

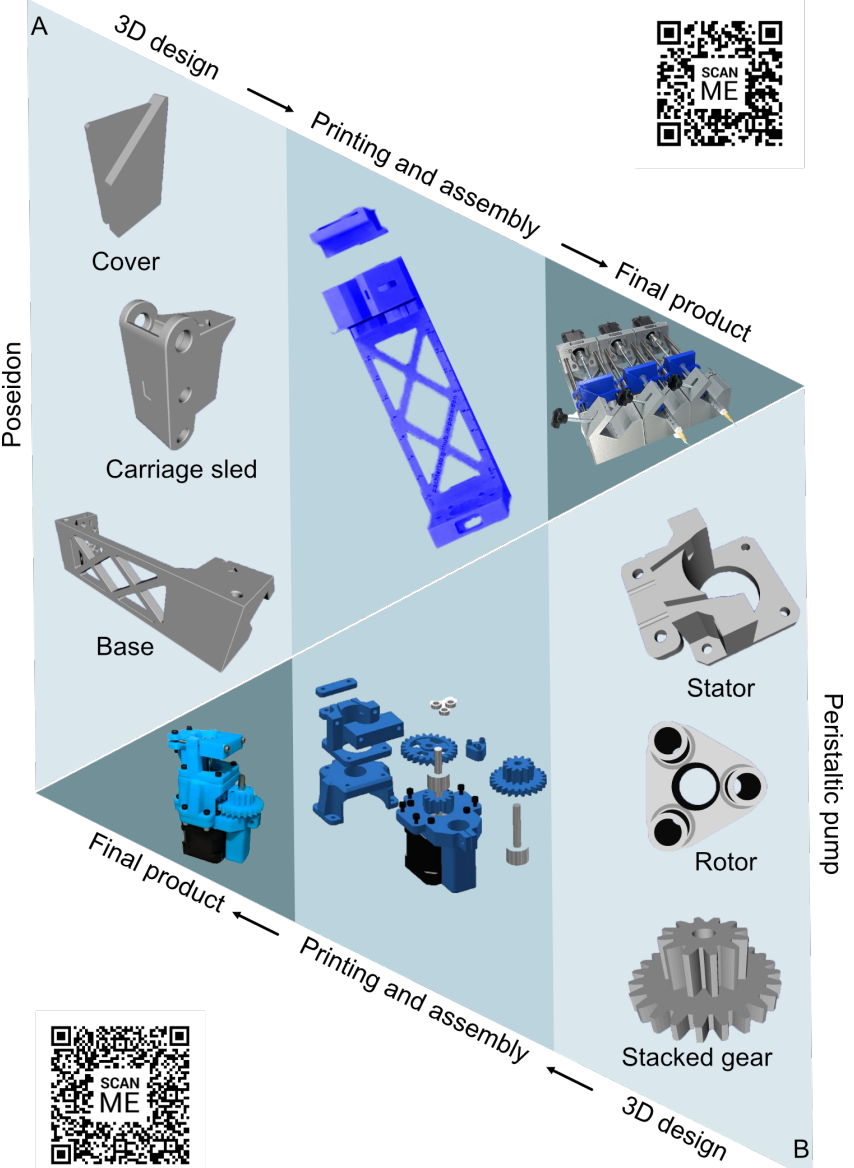

Fig. 4. 3D printed parts for fluidic control. (a) "Poseidon": a syringe pump-based system that supports multiple pump arrays (25). (b) Peristaltic pump system (57). Both systems require an external power source and can be controlled using an Arduino board.

valves (56). Another method to control microfluidics is using pumps. Syringe pumps are simple and can be used to deliver precise volumes of liquids for various research needs (e.g., delivery of drugs to samples during live imaging).

These pumps use a motor that drives the precise movement of the syringe plunger, resulting in accurate fluid volume control. Syringe systems can be paired in multi-pump arrays that facilitate multiple fluids to be pushed in and out of samples. While commercial systems can be expensive, 3D printed options are available that are both highly efficient and highly modular, allowing the addition of more syringe pumps easily (Fig. 4a) $(24,25)$. The highly modular lego-and-3D-printed system NanoJ-Fluidics (aka "Pumpy) is capable of managing up to 128 syringe pumps simultaneously (24). These systems are controlled by Arduino controllers, allowing precise fluid volume manipulation.

Similarly to syringe pumps, fully 3D printed pumps are capable of inducing flow (58). The most common pump is based on peristaltic pumps, usually consisting of three or more valves along the flow channel. 3D printed peristaltic pumps based on planetary gear concepts are effective and can be printed using common FDM or SLA 3D printers (Fig. 4b) (59). These pumps can be employed in microfluidic automation and printed using designs that do not require posterior 
assembly (i.e., "print-in-place") (60). Furthermore, they can also be operated with Arduino controllers, allowing custom flow profiles for handling precise liquid volumes $(57,61)$.

3D printed pumps also are inexpensive to print, with prices going as low as $€ 38$ per channel (61). Peristaltic pumps 3Dprinted using SLA can present durability issues. However, using thermal initiators in the liquid resin and post-processive baking of the component have been shown to greatly improve durability (62).

Some of these pneumatic pumps require complex ancillary control tubing. However, multiplexers allow control over multiple valves employing only a few control channels, thus facilitating the upscaling of designs and adaptation into highly compact and complex microfluidic systems (58). Microfluidic chips can work as sensors, detecting physical and chemical changes inside chambers by measuring volumes on the picoliter scale (63-66).

Microfluidics devices are currently fully 3D printable (67) with the caveat of not being able to provide transparency (58). Printing glass-like transparent material is still not fully attainable and severely limits their microscopy applications. To circumvent this issue, certain parts, such as valves, can be 3D printed and then combined with glass or other transparent materials such as glass or polydimethylsiloxane (PDMS). Here, for example, 3D-printed scaffolds made of ABS or water-soluble polyvinyl alcohol (PVA) filaments have been used. A mould is then used to cover the scaffolds with PDMS. As soon as the PDMS has hardened, water is used to dissolve the PVA filaments without leaving residues, while ABS can be dissolved by acetone. This approach presents the advantage that it allows to print directly onto the coverslips while also providing transparency, allowing excellent applicability for imaging $(68,69)$. Besides lowering costs, one of the advantages of using $3 \mathrm{D}$ printing when compared to other microfabrication methods is the more complex channel designs that can be achieved, such as serpentine flow channels with cross-sectional areas (67) and the compatibility with additional microfabrication approaches like micropatterning (70). Additionally, by bonding printed channels to transparent PMMA sheets, it is possible to produce highly complex arrays, such as straight, spiral, serpentine, curvilinear, and contraction-expansion (71). In some cases, designs such as T-shape pillars are not possible to obtain via other traditional fabrication methods in a single demolding step, but only by using commercial 3D printers (72).

3D printed microfluidic chips have been used to monitor pathogenic microorganisms. For example, using an ABS polymer and FDM 3D printing, a chip allowing bacterial culturing, DNA isolation, PCR and posterior detection using gold nanoparticle (AuNP) probes as an indicator of Staphylococcus aureus (MRSA) was devised. A colourimetric assay based on the interaction between the MRSA mecA gene and AuNP probes was used to confirm the bacterium's presence in the samples (73). An important limitation of the generation of effective microfluidic chambers on these chips is the dimension of the channels (71). The Miicraft printer was designed with this problem in mind and is capable of printing complete microfluidic chambers for lower prices when compared to other methods. This system was used to create an in vitro model of the circulatory system using a cardiac-like onchip pumping system. This was done using four pumps and passive check valves to mimic the four heart chambers and valves. The process was later validated by emulating normal human left ventricular and arterial pressure profiles (74). Microfluidics chips are perhaps the most promising in the field of "organ-on-chip" research, where whole organ-like functions are mimicked using a combination of cell types growing on chambers and channels inside the chip. For example, 3D tissue-engineered skeletal muscle (TESM) cultures have been established using commercial 3D printers by using PDMS to create chambers where cells can grow. Once the 3D cell cultures were established in the chamber, a suspension of hiPSC-derived myogenic progenitors and biocompatible hydrogels made of fibrin and Matrigel (75) was introduced. Differentiation resulted in the successful formation of a TESM culture with a comparable composition to other cultures, including myofibers (72). In addition, organ-on-chip managed to mimic liver interstitial structures containing endothelial cells and primary hepatocytes (76), produce a drug screening assay to assess cell reaction (77), and test for injury (78), hepatitis B infections and even alcohol-driven injury (79). Organoids for live imaging have also been developed in microfluidic chips combined with a bioreactor. The bioreactor provides ideal conditions to grow cerebral organoids to image the organoid self-assembly dynamics without transferring or disturbance. This approach facilitates examination of the sample while also allowing the delivery of drugs to modulate organoid behaviour (80) Other organs have also been modelled, such as the lungs $(81,82)$, kidneys $(83,84)$, intestine $(85,86)$ and heart $(87,88)$.

Biological cages and direct sample manipulation. Sample holders and biological cages that are in direct contact with living organisms are critical imaging components. 3D printed parts used for these applications require biocompatible materials that do not alter the physiology of the cells. While many strategies to ensure biocompatibility exist, generally using ethanol washes together with epoxy resin appears to provide the best results, even when using clear materials (89). Depending on the sample holder's nature or biological cage, autoclavable material might be useful for priorand post-sterilisation during experiments. Structures with these characteristics require printing with advanced plastics that provide high steam, temperature and physical resistance $(90,91)$. More information on material properties and their biocompatibility can be found in Table 2 .

An advantage of using customised sample holders and biological cages is the possibility of tailoring them to fit different imaging platforms. Microscope parts are often expensive and incompatible between different manufacturers or machines. This limits for example cross-instrument compatibly cuttingedge microscopy facilities, where it is common to find many different microscope types. To solve this, the UniverSlide project created a multi-stage, sample biological chamber that can act as a holder or cage for specimens, allowing the growth 


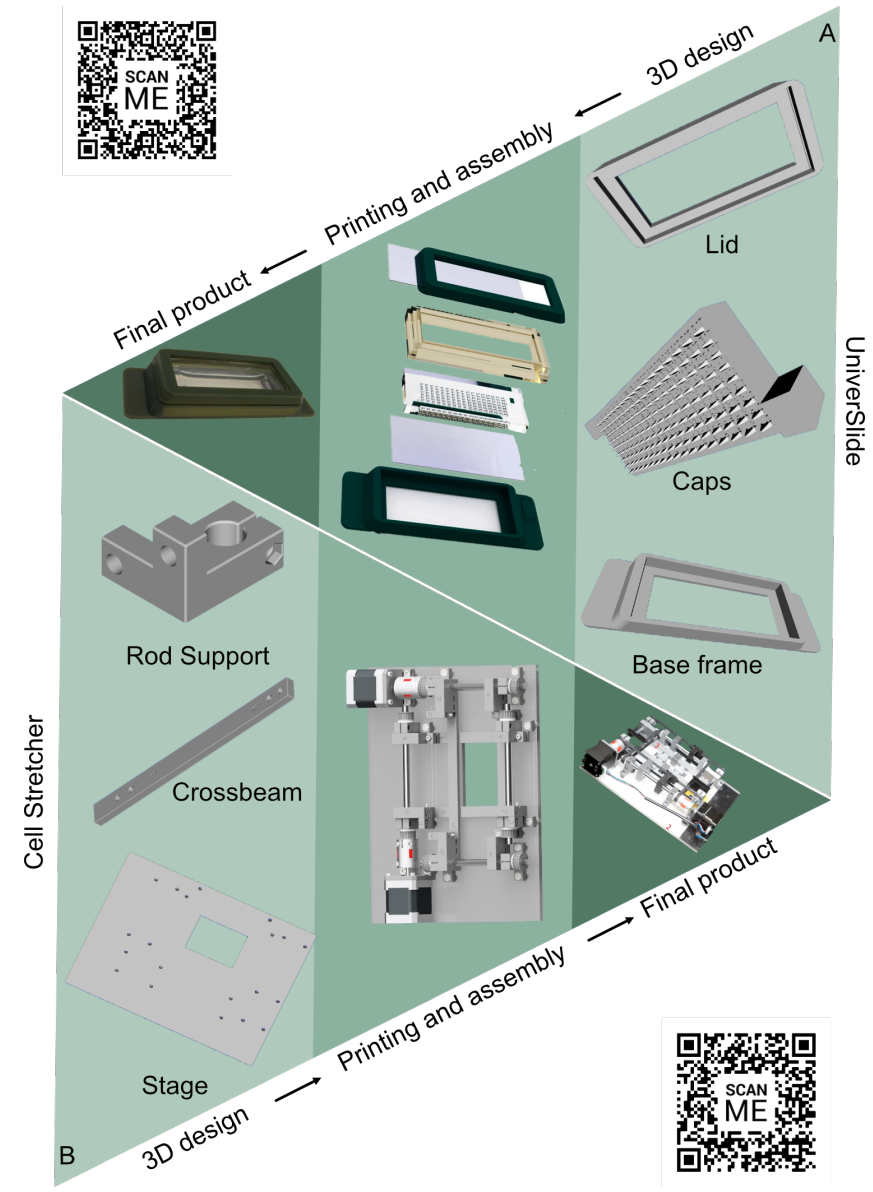

Fig. 5. 3D printed parts for sample holders and manipulation. (a) The UniverSlide is an all-in-one 3D printed microscopy chamber for multidimensional imaging (92). (b) Cell stretcher used to assess tensile properties with controlled cell stretching. This open-source system is directly mounted on the microscope stage allowing live fluorescent cell imaging (93)

of living tissue and easy adaptation between microscopy systems (92). This versatile sample chamber was 3D-printed using SLA and a biocompatible HTM140 resin from Envisiontech (Fig. 5a). The authors agreed that the system can be adapted to use any standard resins since glass and PDMS are the only materials in direct contact with the sample.

UniverSlide was devised to have the dimensions of a regular microscope glass slide (e.g., 26 x $76 \mathrm{~mm} 2$ ) and uses five main parts that include the 3D printed chamber frame, a bottom coverslip, an agarose pad, a PDMS seal and a 3D printed lid with a glass slide (Fig. 5a). The sample chamber can then be filled with cell culturing medium and used for microscopy applications in unicellular and multicellular samples. Also, it is compatible with live and fixed samples (92).

This type of sample chamber can also be expanded to aid experimental procedures. For example, a low-cost cell growth chamber capable of electrical or chemical stimulation of the sample has been devised. Electrical stimulation in mammalian cell cultures is used to assess physiological mechanisms, generally in neuronal cells and myocytes. They are also used to time-resolve intracellular calcium concentrations as a direct result of inducing membrane depolarization (94). Direct sample manipulation is also possible, for example, us- ing tension and other mechanical stimuli to determine tissue properties. This process is achieved using devices that stretch the cell/tissue sample in a controlled manner and measure mechanical properties while monitoring cellular changes using fluorescence microscopy. Commercial cell stretchers are available (95) but are often expensive and hard to customize. The 3D-printed Open source Biaxial Stretcher (OBS) was developed for this reason, improving accessibility to researchers. Additionally, it is compatible with upright and inverted fluorescence microscopes and can perform up to 4.5 $\mathrm{cm} \mathrm{XY-stretches} \mathrm{using} \mathrm{an} \mathrm{electronic} \mathrm{controller} \mathrm{(Fig.} \mathrm{5b)} \mathrm{(93).}$ Another cell manipulation method is Atomic Force Microscopy (AFM), which uses a cantilever that provides data on cellular characteristics such as adhesion strength, elastic modulus, and mechanobiological properties (96-98). AFM can also be combined with microfluidics (99), allowing accurate force control and fluid manipulation inside cells. This type of setup uses an AFM cantilever with an aperture at the tip and an internal channel. This microfluidics cantilever was made using SLA and two-photon polymerization (2PP printing on a SL-printed fluidic interface). This type of cantilever probes provide microfluidics with AFM functionality and are useful for precise fluid manipulation inside cells via cell puncturing (100).

\section{D printing resources and technology in mi- croscopy applications}

This review aims to enable researchers who are unfamiliar with 3D printing to implement of this approach for their own microscopy projects by providing a comprehensive guide of available resources and technologies, including a quick start guide (see "Box 3. How to 3D print" on page 16)

Databases for 3D parts. 3D printing databases exist for both research (e.g., NIH Exchange: a database of 3D printed parts) and more general applications (e.g., Thingiverse), providing $3 \mathrm{D}$ printing enthusiasts the means to obtain complete 3D models. However, a complete database focused on microscopy projects does not exist. This represents a substantial obstacle for newcomers, as information is not centralised and can be difficult to locate because it relies on previous literature knowledge. Therefore, we compiled a 3D printing database for microscopy applications which can be found as supporting information. .

Commonly used 3D printing technologies. Additive manufacturing comprises a variety of different technologies. Although the technology itself is widely known and highly advertised, researchers unfamiliar with 3D printing technology still struggle to fully realise its potential beyond a mere curiosity. 3D printing technology has seen a sharp increase in accessibility as the technology becomes less expensive, with many commercial options offering basic printers for less than $€ 100$ (9). From these widely available printers, FDM and SLA stand out as the simplest and most inexpensive options for a beginner and thus, will be the focus of this review. 


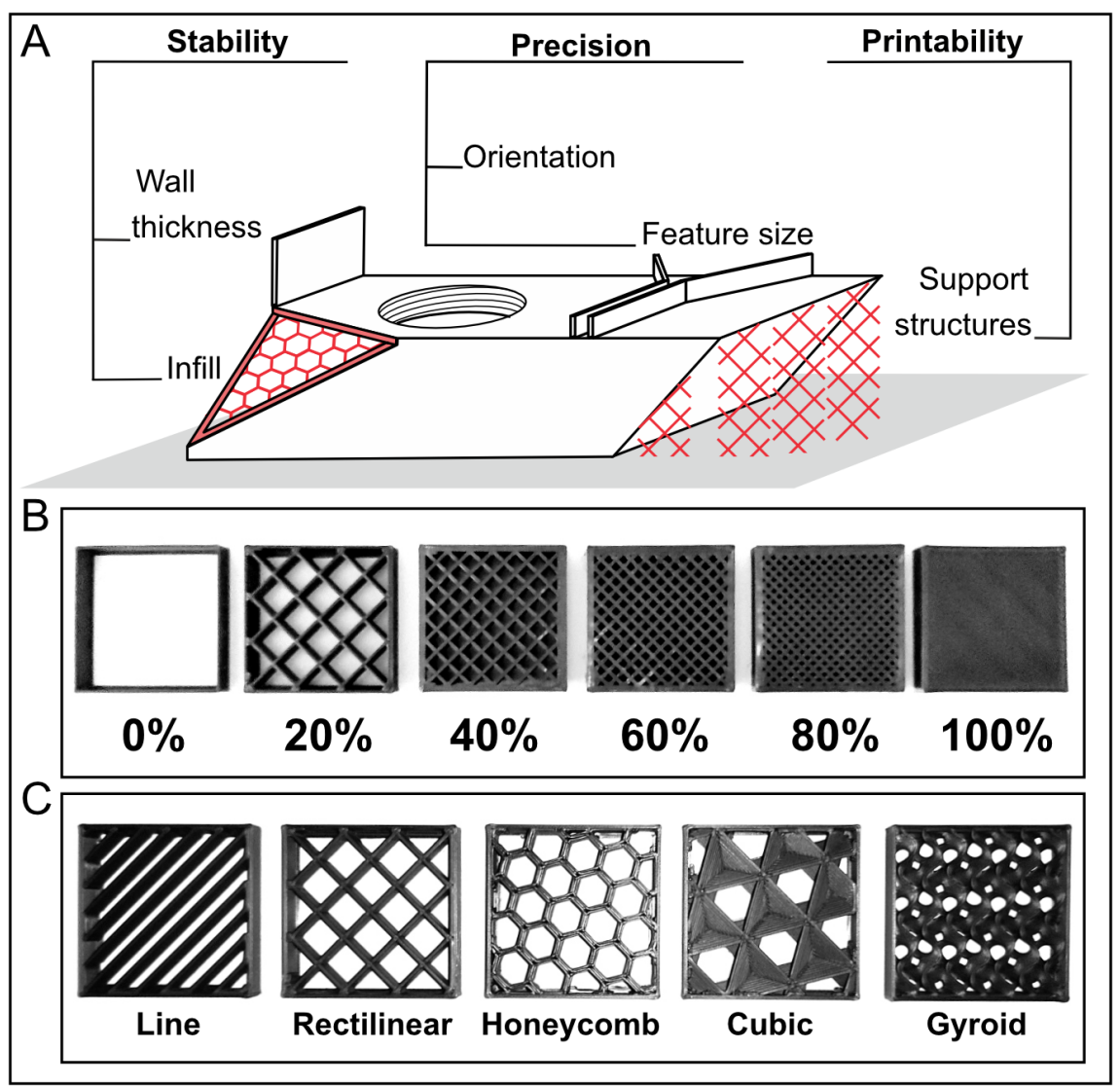

Fig. 6. 3D design principles to ensure mechanical stability, precision, and printability. A) The overall mechanical stability of a 3D-printed object is mainly influenced by the thickness of it's walls and the properties of the infill (e.g., density and pattern). A high printing precision can be achieved by optimizing the print orientation, while the minimum feature size that can be achieved with the printing approach and material has to be considered. Some features, such as overhangs and bridges, might require the implementation of support structures to be printable. B) Examples of different infill densities using the "rectilinear" pattern. A higher infill density contributes to the object's structural integrity but increases the printing time and material consumption. C) Examples of different infill patterns printed with a density of $20 \%$. "Line" and "rectilinear" patterns are the strongest against forces applied in the direction of their main axis, but weak against forces in other directions. Conversely, the "honeycomb" pattern is the weakest against forces applied in any specific direction but the strongest when forces are applied in more than one direction. Several other patterns that explore the three-dimensional space in different manners also exist.
Fused Deposition Modeling (FDM) is perhaps the most popular and cost-efficient of all 3D printing technologies. It is versatile and flexible, with many different materials available to suit specific needs. The most common materials used in FDM are thermoplastics, but composites of thermoplastics and ceramics or metal powders are also available. During the $3 \mathrm{D}$ printing process the plastic material is extruded through a heated nozzle along a predefined path and deposited layer by layer to materialise the design. The printer itself consists of a platform, the print bed where the layers will be deposited in a semi-solid state, a print head composed of a heating block and a nozzle, electric motors that move the print head, and the filament spool holder. Once the g-code of the design is loaded into the printer, a 3-axis system controls the print head, moving in the $x-y$ axis to deposit a layer with paths covering the shape of the initial slice of the 3D design. This is followed by the head or the print bed moving in the $\mathrm{z}$-axis according to the layer thickness, and the addition of another layer using $\mathrm{x}-\mathrm{y}$ axis movements as before. This process is iterated until the final 3D model is recreated. Due to its simplicity and fast turnaround, FDM excels at rapid prototyping. However, the printed structure's quality depends on a multitude of factors, including the material used. A wide variety of materials is available, of which the most common are acrylonitrilebutadiene-styrene (ABS), polylactic acid (PLA), Polyethilene Terephtalate Glycole modified (PETG) polycarbonate (PC), polyamides (PA), and Polypropylene (PP). Additionally, FDM enables colour printing if filaments with different pigments are used.
Furthermore, other elements besides the materials used contribute to the integrity and properties of the prints (Fig. 6a). Several studies have experimented with path-planning and part-orientation to alter the anisotropic mechanical properties of the 3D printed parts (101). The quality of the layerto-layer binding is also crucial as voids forming between the layers reduce the object's strength. An approach to improving these properties is developing composite materials exhibiting higher mechanical, electrical, and thermal properties. These materials are produced by combining the base polymer with fillers. Another approach to increase structural integrity that is highly discussed in the $3 \mathrm{D}$ printing community is infill modulation. Different infill densities can create objects ranging from completely hollow ( $0 \%$ infill) to completely solid (100\% infill) (Fig. 6b). Importantly, this choice influences the weight of the final object and the printing time substantially. Furthermore, the geometry of the infill pattern chosen is also important. For example, a study found that the "rectilinear" pattern resulted in the highest tensile strength than the other patterns evaluated (102). However, while "rectilinear" excels at resisting forces applied in the direction of the pattern's lines, it is extremely weak against forces applied in other directions. Thus, a better choice might be the "cubic" or "honeycomb" patterns, which are less resistant than the "rectilinear" to forces applied in a specific direction but stronger in the others (Fig. 6c). Despite the many advantages of FDM compared to other 3D printing modalities, this approach's nature entails certain caveats. FDM is prone to defects and printing artifacts that impact the printed object's 
A
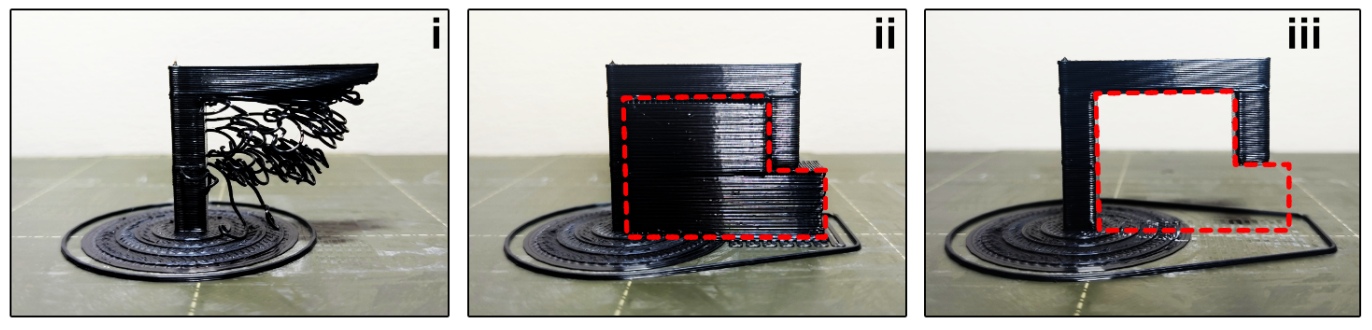

B
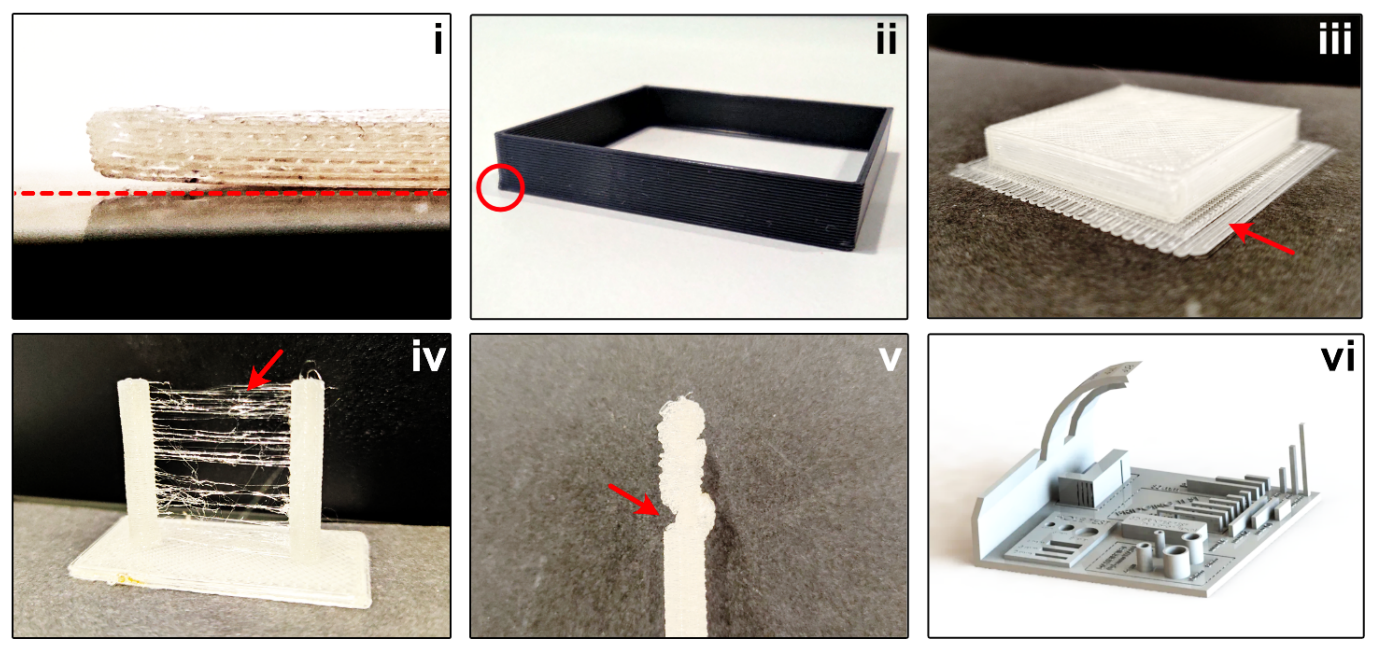

Fig. 7. 3D printing limitations, defects, and artifacts. A) Models containing overhangs require support structures to be printed. (i) Attempting to print an overhang without support structures results in defective prints because the deposition of material requires a platform. (ii) Simple support structures (highlighted by a red box) can be included during the "slicing" process and are printed as part of the object. (iii) After printing is finished, the support structures can be removed, and the final shape is achieved. B) FDM is prone to several printing defects and artifacts, which can result from a multitude of factors, such as the thermodynamics of the printing environment (e.g., cooling efficiency). (i) "Warping" or "curling" is a deformation resulting from the materials' expansion coefficient. It produces a defective object and can lead to catastrophic failures. The red dashed line depicts the printing plane and highlights the degree of deformation. Adequate adhesion and the use of a heated printing bed can help avoid this effect. (ii) Conversely, high bed temperatures can lead to "Elephant's foot", which consists of a first layer that is wider than the subsequent layers. (iii) Printing a raft can help avoid "warping" by increasing adhesion to the printing bed. It can also prevent "elephant's foot" by re-directing the heat-induced deformation from the object to the layers added to the bottom of the object. (iv) "Stringing" or "oozing" consists of thin filaments bridging different structures and results from material extrusion in between movements of the printing head. It can be reduced by increasing printing speed, tuning of the extrusion/retraction cycles, but can also be removed posteriorly using a hot-air gun. (v) "Layer shifting" typically occurs when the printing head collides with the object during printing, resulting in a chronic printing offset that misaligns the layers printed after the collision. (vi) Several printing settings can be tuned to achieve a perfect printing job. The tuning procedure(s) are now quicker and easier thanks to contributions of many model creators who upload "tuning models" to 3D model online databases. Some models are directed towards a specific tunable feature, while others, such as the one depicted, combine all the tunable features in a single object (https://bit.ly/3tx6Oag).

aesthetics and practical applications. For example, objects containing overhangs with more than 45 degrees require the use of supports to be printed correctly (Fig. 7a). These are often removed manually but can also be removed chemically if special dissolvable materials are used to produce them, the most common being the water soluble PVA. The support removal process results in rough surfaces that require sanding or polishing to be smoothened (103).

Several printing artifacts can result from a multitude of factors (Fig. 7b). "Warping" is a curling deformation often accompanied by a partial or complete detachment of the object from the printing bed (Fig. 7bi). It can result in catastrophic failure if the deformation causes the object to intercept the nozzle's path. Warping occurs as a result of the materials' expansion coefficient. When the material is melted before extrusion, it first expands and then shrinks slightly when it cools down. Thus, warping is more common in materials with higher melting temperatures (e.g., ABS), and it can be minimised by controlling the printing environment, for example, using heated printing beds and enclosed build chambers.
Conversely, a high bed temperature and insufficient cooling can result in a first layer that is slightly larger than the subsequent layers, an effect known as "elephant's foot" (Fig 7bii). This deformation is especially problematic in objects that require a precisely defined shape, such as a part intended to fit a tight slot. Elephant's foot is more frequent in larger prints, in which the weight of the object presses down on the partially-cooled first layers. A nozzle positioned too close to the printing bed may also generate this effect by forcing the extrusion of material beyond the predicted line width. Thus, "elephant's foot" is typically solved by adjusting the printer's settings and environment. Another solution for warping and "elephant's foot" is printing a raft, which is an additional and wider first layer meant to be damaged in place of the original first layer (Fig. 7biii). Similar to supports, rafts need to be removed after the printing job is finished, resulting in rough surfaces that might require post-processing.

Another common printing artifact is "stringing" or "oozing", which happens when material is extruded while the nozzle is moving to a new location (Fig. 7biv). Consequently, thin 
Table 1. Commonly used 3D printing technologies for microscopy applications. Fused deposition modeling (FDM) and Stereolithography (SLA) are two 3D printing technologies with different applications. FDM is generally more suitable for inexpensive, rapid prototypes with modest structural complexity and substantial mechanical properties, while SLA is more suitable for highly intricate objects where high mechanical impact is not present.

\begin{tabular}{|l|l|l|}
\cline { 2 - 3 } & \multicolumn{1}{c|}{\begin{tabular}{c}
\multicolumn{1}{c|}{ FDM } \\
Fused Deposition Modelling
\end{tabular}} \\
\hline Technology & Filament deposition by thermoplastic extrusion & Sterlithography \\
\hline Materials & PLA, ABS, PLA, PETG & Resins \\
\hline Costs & Relatively low & SLA higher than FMD, (m)SLA comparable to FDM \\
\hline Pros & Affordable materials, printing of multiple colours and materials & Finer structural detail and complexity, quiet operation \\
\hline Cons & Support structures required for complex geometries & Limited choice of materials, untidy, requires postprocessing \\
\hline Applications & Rapid prototyping, Optomechanic parts & Cast for PDMS chambers \\
\hline
\end{tabular}

strings of plastic are left behind. Possible solutions to overcome "stringing" are increasing the speed at which the extruder moves to reduce the time during which material is extruded between movements, or increasing the speed and length of "retraction", where the filament is pulled back before moving the nozzle in a new position. In contrast, increasing the movement speed past the capabilities of the printer's motors might result in material extrusion before the nozzle reaches the desired position. Thus, the layer(s) printed will be misaligned with the previous layers, causing a catastrophic artifact named "layer shifting" (Fig. 7bv). Tuning a 3D printer to avoid these and other printing artifacts is now easier due to the availability of several 3D models that can be used to optimise specific printing settings. Some tuning models are directed towards tuning a specific aspect, while others are "all-in-one" models that combine multiple features of 3D printing (Fig. 7bvi).

Stereolithography (SLA) is the oldest form of 3D printing (104). As it is a highly versatile and accurate form of $3 \mathrm{D}$ printing, although beeing more expensive, the intricate details it can produce are fairly superior to the FDM. Instead of using thermoplastics like FDM methods, it uses thermoset liquids -in the form of liquid resins- cured by UV light. Here, UV light selectively illuminates a small liquid resin area, triggering initiators and photo-polymerising it via radical polymerization, an exothermic process (105). The polymerisation triggered by these initiators creates covalent bonds between the liquid resin monomers. Two transition states occur during this light-based curing process: gelation, where the material transitions from liquid to rubber, increasing its viscosity; and vitrification, where the rubber-like material transitions into a solid resin (105). This process is done in layers but due to the use of resin materials, the material's physical properties such as tensile strength and flexibility are usually inferior when compared to FDM. To overcome this limitation, material manufacturers are constantly creating new formulations and, in some cases, were able to create resins presenting char- acteristics that are comparable or even better than FDM materials in terms of flexibility or hardness, heat resistance, solvent resistance. Due to its unique printing method, SLA can confer anisotropic properties to the printed materials and provide the highest possible resolution, accuracy, and smoothest surface of all 3D printing technologies. For this reason, SLA is widely used in the industry to create prototypes with intricate patterns, casting, and moulding. SLA printers usually use a laser for curing the resin. This process is, however, slower and more complex than FDM printing. In addition to this, the layers of the SLA print can go as low as $25 \mu \mathrm{m}$, making the printing process slower than a standard FDM. In recent years, masked SLA (mSLA) printers have reached the consumer market. If the chemical process is similar to the SLA -crosslinking resin using UV light- the way the light is processed is different. Whereas in the SLA, a laser is employed, in mSLA a set of UV LEDs is used for the crosslinking process, and an LCD on top of them will control where the UV light passes, effectively using the transparent or black LCD pixels as mask lithography. Thanks to this ingenious method, those printers are equally or even more inexpensive than) FDM printers, and, as they cure one complete layer at a time without the need of moving a laser (or a printhead), they are also faster than SLA or FDM. The inexpensive availability of those printers, together with their speed and precision may open the possibility of using mSLA printers in the lab (72). Both FDM and (m)SLA technologies are usually compared based on availability, cost, the durability of the materials, and the level of structural details provided (see Table $1)$.

Materials for 3D printing. Besides the printing technology and the 3D-design, one crucial factor defining the quality and physical properties of the 3D-printed component is the material choice. Thus, a lot of effort is taken to optimise material composition to increase the printability and print quality, but also to enhance physical properties like strength, flexibility, and biocompatability (106), or optical properties (107). 


\section{Box 2. FDM-material guide}

The thermoplastic ABS convinces by its low cost, easy processing, and high mechanical and chemical stability and is widely used for rapid prototyping (108). Parameters such as infill density, layer thickness, orientation, raster angle, and air gaps are essential to provide strength to the printed part (109).

PLA is a thermoplastic with a lower impact strength than ABS but better overall tensile strength. The tensile strength is dictated mainly by the raster angle, width and layer height. Reducing the layer height is necessary to avoid formation of voids and improve the mechanical integrity of the structure $(110,111)$.

Nylon PA presents high chemical resistance, tensile strength, and flexibility. These mechanical properties even improve at higher temperatures $(112,113)$.

PET has good chemical and impact resistance. This clear thermoplastic is commonly used in disposable plastic bottles and packaging since it is non-toxic and biocompatible. (114). PET has the advantage of being a highly recyclable material. However, it is brittle, making PETG the candidate of choice when PET plastics are needed $(114,115)$.

PETG is a copolymer formed by Polyethylene Terephthalate (PET) and Ethylene Glycol. It is a high impact and chemical resistant thermoplastic that can be recycled (115). It is also transparent and biocompatible. However, its low resistance against ultraviolet (UV) light results in discolouration and brittleness when exposed to it for long periods (116).

PC is a thermoplastic that presents high durability, impact and heat resistance. The material is also moderately flexible and transparent but difficult to print as it requires high temperatures to be extruded properly, resulting in cooling difficulties and proneness to warping (117).

PEEK, PEI and PPSU are high-performance polymers providing unrivalled mechanical, chemical, and thermal properties. It is commonly used in industry for highly wearable parts such as those found in aircraft, cars, drones and rockets (118) or in biomedical applications $(90,91)$. Due to higher printing temperatures, specialised printers are required (119).

TPE is a flexible rubber-like combination of elastomers and polymers that are also recyclable. It is highly flexible and soft, with particularly good impact resistance and shock absorption (120).

Composite materials contain supplements such as metals (e.g., copper and iron) or carbon-based elements (graphene or short carbon fibres). Supplementing with iron improves thermal conductivity, storage modulus, and glass transition temperature (121). Short continuous composites show increased rigidity and strength, corrosion resistance, and improved chemical resistance $(122,123)$. Among these elements, carbon, Kevlar and glass are also used alongside naturally found components such as basalt, jute and bamboo $(124,125)$.
FDM materials. FDM uses thermoplastic melts dispensed by a heated extrusion print head. Polymer filaments are available commercially and require a balance of processing temperature, build speed, polymer melt rheology, and CAD shape parameters. FDM has been used to print polymers, polymer matrix composites (PMC), biocomposites, polymer ceramic composites (PCC), and fibre reinforced composites (FRC) (126). Given its wide appeal, FDM uses a large selection of thermoplastics commercially.

Common materials include standard plastics used for noncritical functions and engineering plastics that, due to their mechanical properties, are suitable for high mechanical stress work. The most prominent materials are Acrylonitrile Butadiene Styrene (ABS), Polylactic Acid (PLA), Acrylonitrile Styrene Acrylate (Nylon PA), Polyethylene terephthalate (PET), Polyethyleneterephthalate Glycol-modified (PETG) Polycarbonates (PC), and polypropyene (PP). The best starting materials for 3D printing are PLA and PETG for their simplicity of use and relatively low printing requirements. Most of these materials are well suited for general use due to their low melting temperature and cost. More advanced thermoplastics, known as high-performance polymers due to their improved chemical, thermal and mechanical properties, are also available at much higher costs and should be used in critical applications. These polymers include Polyether Ether Ketone (PEEK), Polyetherketoneketone (PEKK) and Polyetherimide (ULTEM).

A detailed description of the properties and applications of the filament materials used in FSM is provided in the "Box 2. FDM-material guide" in the right column.

SLA-materials. Photocuring 3D printing methods use a small dose of energy in the form of light to trigger covalent crosslinking of the material. For the covalent crosslinking to occur, three key components are needed: the initial energy (light), the printing platform, and the photocurable resin serving as the base material. Decreasing the materials' energy requirement or increasing the printer's energy output results in faster printing, with the former option being the main focus of optimisation (9). Since light can only print a layer of limited thickness, multiple light exposures are needed (127).

From the chemical perspective, the process of gelation results from the crosslinking of the photoresin's monomers. In this process, a light source functions as an energy initiator that triggers a photoinitiator (PI), resulting in polymerisation. These PIs are usually single molecules that cleave radical fragments when exposed to the light of a specific wavelength (127). Common PIs include phenylphosphine oxide (e.g. Irgacure) (9) and Acyl phosphine oxides (e.g., TPO and BAPO) (106). Other methods use two elements: a lightabsorbing molecule and a co-initiator (106).

Furthermore, PIs, such as ethyl 4-dimethylaminobenzoate (DMAB) and zinctetraphenylporphyrin (ZnTPP), use visible light to achieve the same effect but display high toxicity $(128,129)$. However, other PIs have been used with better results in biocompatibility, such as 3-hydroxyflavone (3HF) 2018, which displays overall lower toxicity allowing biomedical applications. Another important aspect of 
SLA are the monomers used to form the 3D object. Originally, they were composed of combinations of diacrylates dissolved in liquid acrylate or methacrylate (9). Many types of monomers are used in commercial and research applications; a few reviews can also be consulted for more in-depth information $(9,131,132)$.

Commercially speaking, many different light-curing resins are available with different prices and properties. However, many of these resins are proprietary, making it difficult to understand the material's nature. Many commercial resins that mimic other plastic materials are sold as-is, often using their characteristics and similarities with existing polymers instead of disclosing their chemical contents. For this reason, in this review, resins are classified by their function and how they can be found commercially, without mentioning specific brands. Standard resins are the cheapest available and are very suitable for visual applications because they provide a smooth surface finish and a high printing detail level. Their main drawback is brittleness, which makes them incompatible with parts that suffer mechanical stress. Clear resins are helpful when transparent materials are needed but require additional post-processing to achieve a clear glass-like finish while suffering from brittleness.

Castable resins are helpful in creating mould patterns and provide very high printing detail. Tough resins mimic the mechanical and chemical properties of ABS or PLA, they are often slightly worse than their thermoplastic counterparts and don't provide the same thermoresistance properties. Temperature-resistant resins, on the other hand, are suitable for thermal applications and moulding but generally expensive. Dental resins are usually used in biomedical settings and provide the best biocompatibility while maintaining good mechanical properties and high abrasion resistance. However, they are costly and for microscopy applications, postprocessing to increase biocompatibility is a viable option, as mentioned in the next section. The properties and suggested applications of these materials are summarised in Table 2.

\section{Current 3D printing challenges and limita- tions}

3D printing presents ample advantages in customisation, design freedom, costs, accessibility, and the capacity to produce highly complex structures compared to other (micro)fabrication methods. However, these advantages are accompanied by drawbacks and challenges, including: Limited high-throughput application, low mechanical and anisotropic properties, printing errors, and limitations on material choice. Additionally, it requires knowledge of 3D modelling and understanding the materials, and the resolution needed. For projects involving direct contact with living organisms, it is important to take the material biocompatibility into account. Many of these materials can be highly toxic to cells and whole organisms (133). Toxicity can be avoided in any case, by using biocompatible post-processing methods if needed. Beyond the biocompatability aspect, there is an increased concern about the potential environmental impact caused by 3D printing, as the process consumes large amounts of en- ergy, produces plastic waste, and generates air pollution.

Fabrication. The successful design and execution of a 3D printing project can be challenging, as various unexpected and unwanted defects in the object shape can emerge as result of the fabrication process. 3D printed parts often carry artefacts depending on the object's geometry, such as in the case of an insufficient polygon-approximation of curved surfaces (103). Post-processing can often alleviate these defects but good design practices are required to minimise them. Therefore, for the 3D design, it is important to optimize the printing orientation, create supporting structures when the design requires it, and add enough layers in the slicing step.

The "layer-on-layer" nature of FDM printing can be a limiting factor in certain designs. For example, some designs contain features called "bridges", requiring horizontal material deposition between two raised points (Fig. 8a). Printing perfectly horizontal bridges is often required. However, since FDM requires the printing material to be melted at high temperatures and then hardened by cooling, the bridge layers tend to become deformed due to gravity between these two stages. Thus, "bridging" can be troublesome and sometimes even impossible to achieve. Naturally, common strategies to improve "bridging" include increasing cooling, decreasing the rate of material extrusion, decreasing nozzle temperature, and decreasing printing speed.

Furthermore, adding support structures to the design is a common approach to circumvent this limitation. However, these structures need to be removed after printing is finished, resulting in increased post-processing times. Also, certain features are too structurally complex to include support structures or to allow their removal without resulting in substantial deleterious effects. An interesting example of how the inclusion of support structures can be avoided by design is the OpenFlexure microscope base. In this model, an elevated platform needs to be printed between four columns without directly contacting them. The model becomes printable without support structures by including a bottom layer that bridges the closest points between the columns, upon which another bridging layer is printed in a different orientation. This last layer serves as the floor on which the elevated platform is printed (Fig. 8bi-iv).

An important challenge during printing is the formation of voids between material layers. During printing, layer deposition can create unwanted porosity. This porosity often reduces the printed object's mechanical properties (134), and this is influenced by the choice of material and technology used, with FDM having void issues more commonly than SLA (134). In the case of FDM, reducing porosity during printing requires an increase in the printed object wall thickness. However, this approach also reduces the final product's tensile strength alongside further design issues (135). Interestingly, this 3D printing flaw can also be exploited by controlling the porosity to develop porous scaffolds that can be employed in tissue engineering applications (136).

The mechanical properties and anisotropy of structures also present a challenge, as each printed layer is not the same as the one before. This property often results in unwanted 
Table 2. 3D printing materials and limitations. 3D printing has multiple advantages and disadvantages depending on the technology and materials used. Most importantly, these limitations can be circumvented by an appropriate understanding of the limitations of the materials to correctly apply them.

\begin{tabular}{|c|c|c|c|c|c|c|c|c|c|c|}
\hline & Material & Speed & Low cost & $\begin{array}{l}\text { Heat } \\
\text { resistance }\end{array}$ & $\begin{array}{l}\text { Chemical } \\
\text { resistance }\end{array}$ & High strength & Flexibility & High detail & Transparency & $\begin{array}{c}\text { Bio- } \\
\text { compatibility }\end{array}$ \\
\hline \multirow{6}{*}{ FDM } & ABS & 0 & 0 & & & & & & & $\left.{ }^{\star}\right)$ \\
\hline & PLA & 0 & O & & & & & () & & \\
\hline & PETG & (0) & 0 & & & & & & & \\
\hline & PEI, PPSU \& PEEK & & & 0 & 0 & & & & & \\
\hline & Nylon PA & & & & (0) & o & 0 & & & \\
\hline & TPE & & & & & & 0 & & & \\
\hline \multirow{8}{*}{ (m)SLA } & Standard resin & & 0 & & & & & (0) & & \\
\hline & Clear resin & & & & & & & () & 0 & \\
\hline & Castable resin ${ }^{* *}$ ) & & & & & & & 0 & & \\
\hline & Rubber-like resin & & & & & O & 0 & (0) & & \\
\hline & Though resin & & & & & (0) & & 0 & & \\
\hline & Bio-based resin & & & 0 & & 0 & & 0 & & \\
\hline & Thermo resistant resin & & & () & & & & () & & \\
\hline & Dental resin & & & & & & & (0) & & O \\
\hline
\end{tabular}

mechanical behaviour, particularly when vertical tension or compression are exerted on the printed object. This is more common in thermoplastics printed with FDM (137). Another factor affecting tensile strength is the printing orientation. For example, the printing angle presents a relationship with the elasticity of the final product when using Acrylonitrile butadiene styrene (ABS) (138). FDM also results in the appearance of layers in the final printed product. This is perhaps not an important factor when the part is not visible, but the exterior parts often need post-processing, such as sintering, to correct this problem (103). For SLA this is usually not an issue.

Biocompatibility. Regardless of the material and 3D printing technology used, if the 3D printed object will come into contact with biological samples and tissues, particularly in a medical setting, the components must abide by the ISO 10993. This certification comprises several standards for evaluating medical devices and material biocompatibility to assess and manage the biological risk (139). Polymers in contact with biological tissues undergo degradation due to mechanical or chemical stress, especially in the case of long-term contact. Materials in 3D printing applications for microscopy are generally not expected to undergo long term contact with living tissues. Nonetheless, material toxicity needs to be assessed. This review focuses on 3D printing in microscopy applications and thus, its scope is limited to materials that are in contact with organisms, tissues, and cells during imaging approaches. Notably, these include both the biological specimen and the user. Reviews that deal with long-term exposure of 3D printed materials can also be found as part of biomedical research (140-143).
Given that FDM is the most user-friendly 3D printing technology, it is reasonable to adopt this technology for biological purposes. Previous work has employed materials such as ABS (144), PC (145) and PET (146) with little to no toxicity when used along with collagen coatings as a substrate (ABS and PC) or just by washing (PET). Another method tested was UV light treatment, where ABS-like materials were successfully made compatible with zebrafish embryos (147).

On the other hand, photopolymers are often more toxic due to their nature and the potential residues that remain following the photo-curing processes (133). Several studies considered 3D printed objects using SLA technology as toxic when used directly in biological applications without postprocessing (148-150). Accordingly, photoinitiators (e.g. BAPO and TPO) are known to have cytotoxic effects at low micromolar concentrations in human cells, leading to mutations and genetic instability (151). Similarly to FDM-based printed objects, improving biocompatibility requires postprocessing. There are a variety of post-processing methods available which can be adapted depending on the nature of the resin material or its function. One of the reasons that have been suggested to underlie toxicity is the chemical composition of the $3 \mathrm{D}$ printing material itself. Often, the complete formulation of these materials is only known to the manufacturers, but photoinitiators and acrylate monomers are known to be toxic for living organisms (149). Material Residues remaining on the printed objects' surface can be washed with ethanol, sonication, and sterilised with UV light (149). Another study identified uncured residual monomers in the objects' surface using HPLC-MS, explaining their high toxicity, and suggested using a combination of residual photopolymer extraction via supercritical $\mathrm{CO} 2$ treatment and 
A

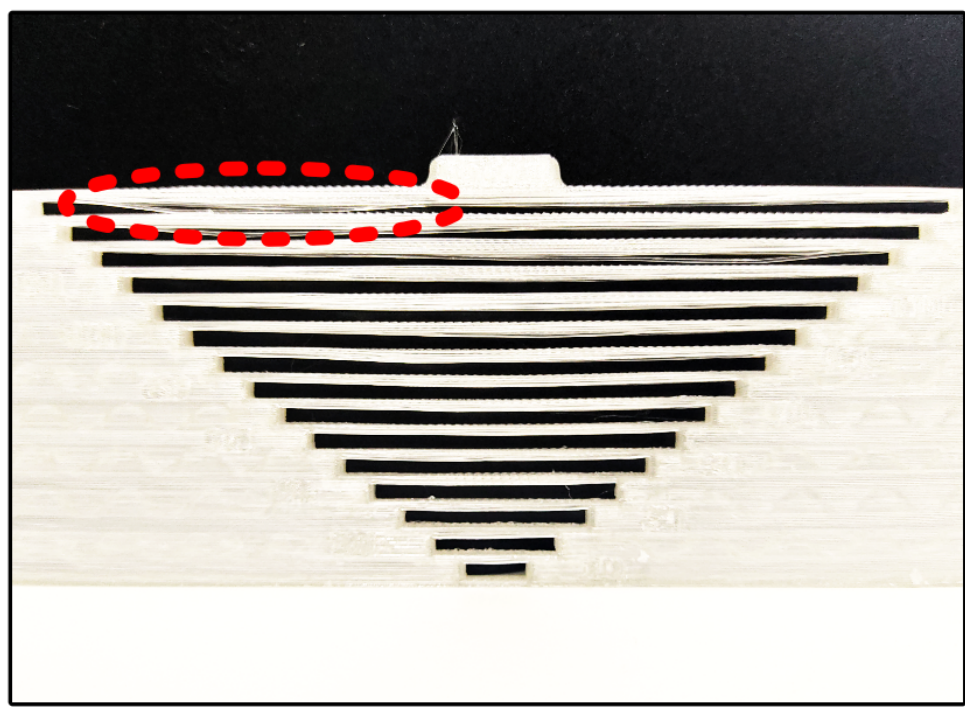

B
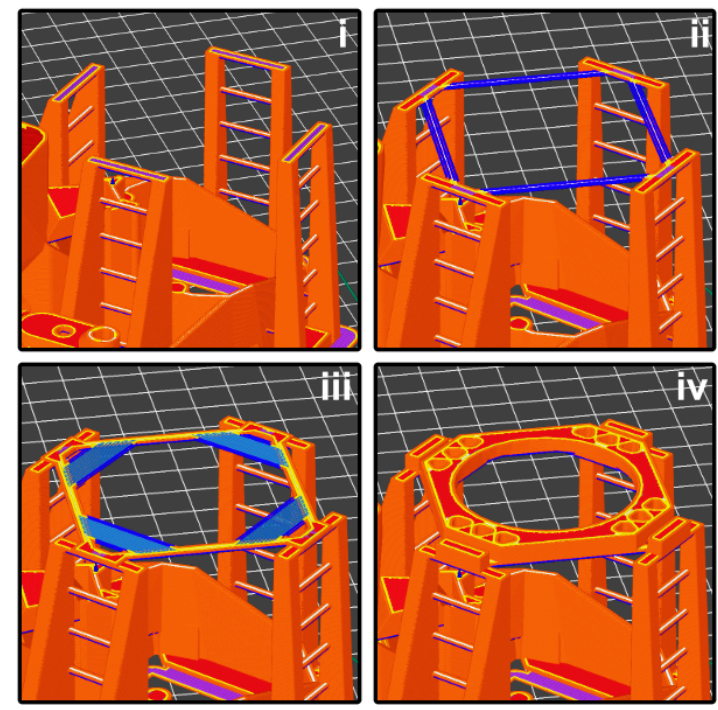

Fig. 8. 3D printed bridges in FDM. A. Printing bridges requires horizontal material deposition between two raised points. "Bridging" can be troublesome because the material needs to be melted at high temperatures to be extruded, and then cooled down to harden. Thus, bridges tend to be deformed due to gravity when cooling is insufficient, or the length of the bridge is large (red dashed box). B. (i) The OpenFlexure microscope base features an elevated platform between four columns that would typically require support structures to be printed. The design employs a "bridging" strategy to avoid the use of support structures. (ii) A "bridging" layer is first printed to connect the closest points between the columns. (iii) A second layer of bridges is then printed in a different orientation. (iv) The last layer serves as a base on which the elevated platform is printed.

post-curing (148). UV light has also been successfully used to reduce toxicity, perhaps by finishing curing any uncured residues (147). Using a nitrogen atmosphere and high temperature is also an effective approach to detoxify material. However, it negatively impacts the material's transparency, making it incompatible with clear/transparent resins (150).

Lately, surface coating with Hydrophobic Epoxy Resins has been shown to improve biocompatibility $(89,152)$. Although epoxy resins require extra steps of preparation, they can dramatically increase the printed object's biocompatibility, up to the standard of commercially available cell culture vessels. These steps include curing the resin by heating overnight at $45^{\circ} \mathrm{C}$ followed by PBS and ethanol washing steps. Epoxy resins are also compatible with transparent materials, making them a suitable candidate to combine with cell culture vessels (89).

Thus, although many 3D printed materials are not biocompatible right after the printing process, most can be made biocompatible through post-processing. Research on these processes requires caution and an additional layer of material and post-processing tests to assess potential undesirable effects in the specimens studied.

Autofluorescence. An important aspect to consider in materials used for microscopy applications is autofluorescence. Autofluorescence is the tendency of a material to emit fluorescence when illuminated at specific wavelengths. This property is problematic in imaging applications because many materials are autofluorescent, and the autofluorescence spectra often overlap with the common spectra chosen to image samples (153). Testing the materials for autofluorescence is a general recommendation for $3 \mathrm{D}$ printing materials used in microscopy applications, to minimize this effect.
Environmental impact. The environmental impact of 3D printing processes is still an ongoing topic of discussion within the field (154). To address the environmental impact of 3D printing, three aspects will be discussed in this review: energy consumption, waste management, and air pollution (155). Energy consumption is considered the factor with the largest impact, particularly in mass production (10). Energy consumption depends on material choice, build volume, layer thickness, and printing speed. The energy consumed during 3D printing can be separated into three phases: preheating, printing, and cooling. Current 3D printers are not highly optimised for energy consumption (156). This optimisation lies partially in the often overlooked cooling systems of 3D printers, which mostly rely on electrically powered fans to dissipate heat (157).

Material waste is also a significant factor in the environmental impact of 3D printing. The two biggest challenges in this regard are the use of recycled materials and recycling waste. Using recycled material for 3D printing is not impossible, as several materials, such as PLA and ABS, can be processed and turned into filaments again (158). Recycled PLA or PETG have also started appearing on the market. Another important piece of plastic, usually overlooked when talking about environmental impact, is the spool holders, where the filament is coiled on. As this is a single-use product, some manufacturers have already started using cardboard instead of plastic for spool holders. As the consumer drives the market, a proper choice of recycled filaments and spool holders may guide the market in a more environmentally friendly 3D printing attitude. In addition to this, from the consumer point, the use of support material should be considered. As this material is thrashed right after the print, it would be environmentally beneficial to design a 3D printed part that would 
use as little support as possible, or none at all. Regarding the recycling of material it has been suggested that a resin identification code should be added to recycling guidelines, particularly as the technology becomes more common in domestic settings (155). For FDM, the recycling process is more straightforward. The material can be processed into small pieces and then reformed into filaments using a heated extruder. The downside is that the heating process destroys the chemical bonds of the polymer, making it weaker. To improve the strength of recycled materials, small amounts of non-recycled material can be added (158).

Finally, indoor air pollution is also a concern in 3D printing. The thermoplastic extrusion process can generate particles of volatile organic compounds (VOC), which can be dangerous to humans and the environment (159). This is particularly hazardous in enclosed environments, and protective clothing and masks are recommended, as VOC emissions are related to the thermal degradation of the polymers and additives used in the 3D printing material. One potential solution is using different low-toxicity thermoplastics such as bio-based ones (PLA), and avoiding fossil fuels produced plastics (ABS) since they require lower temperatures to melt printing, reducing emissions and energy consumption.

In contrast, 3D printing can positively impact the environment, as this technology allows more efficient fabrication compared to traditional manufacturing methods, providing a way to optimise industrial processes. The design of the $3 \mathrm{D}$ printed part is also important, as parameters such as layer thickness or printing orientation can demand more energy, requiring fine-tuning to optimise energy consumption as much as possible. Additionally, using "green" or bioelements as $3 \mathrm{D}$ printing materials can reduce the printing process's environmental impact (160). 3D printing is still a young technology and there is ample room for optimisation, particularly in energy consumption and cooling systems, recycling of materials, and emissions. As the technology becomes more standardised, machine design and printing processes are expected to improve.

\section{Outlook}

Additive manufacturing is revolutionising industry and research, and it will possibly become the technology of choice for manufacturing processes. This review aims to introduce researchers to the advantages and limitations of $3 \mathrm{D}$ printing by giving a wide view of the application of commercial printers. The versatility of additive manufacturing technologies and the relative accessibility will only increase with time, as the field is still in its infancy.

The obstacles that 3D printing currently faces involve the 3D design of objects, available materials, biocompatibility, energy consumption, waste management, pollution, and largescale applications. However, these are minor setbacks and are expected to improve as the technology becomes more common and easier to use. Another limitation lies in the materials used for 3D printing. Fortunately, materials with high mechanical and chemical resistance that are also less toxic are actively researched both in industrial and academic settings.
Box 3. How to 3D print: A quick start guide

This step-by-step guide allows you to get familiar with the basic steps of planning and performing a successful 3D-printing project using FDM, the most common commercial 3D-printing technology. This guide is also available as a video (Movie S3).

Step 1 - Preparations Before you start off, make sure you have everything you need: a computer, a $\mathrm{SD}$ card, a 3D printer, and the printing material. At the time of writing this review, affordable desktop $3 \mathrm{D}$ printers capable of performing most printing jobs are the Creality3D Ender-3 V2, the Original Prusa i3 $\mathrm{Mk} 3$, and the Anycubic Mega. However, care should be taken to ensure that the 3D printer is adequately sized to print the object as down-scaling or printing subsections is not ideal in most cases, and largely defeats the purpose of 3D printing. As mentioned in the previous sections, the choice of material is crucial. The most commonly used materials for FDMbased 3D printers are PLA and PETG. Materials for FDM are sold in the form of rolls of filament at a price of approximately $€ 25$ per kilogram, but prices vary considerably depending on the quality.

Step 2 - Obtaining a digital 3D model The process of 3D printing starts with an idea or the need to produce a specific object. This object has to be represented in a digital format precisely defining the shapes and measurements of the object. You can design a 3D model "from scratch" or by modifying a model previously created using open-source or commercial computer-aided design ("CAD") software. TinkerCAD, for instance, is a user-friendly and web-based platform that can be used even by inexperienced users to design virtually any object. More sophisticated and technical programs also exist, which allow for more precise control of the design's parameters and the production of professional models (see also "Box 1. Software Tools", p. 2). The 3D CAD model is then exported in the STL file format, which encodes the object's surface geometry but has no information about its colour or texture. Alternatively, the CAD and STL files can be directly shared between users, or downloaded from 3D model databases (e.g., https://www.thingiverse.com, also check the "3D-Model Database").

Step 3 - Instructing the 3D printer Once you have created the the STL file, it can be imported into a "slicing" software (e.g., PrusaSlicer or Cura) to generate a set of instructions, called G-code, that can be directly interpreted by the 3D printer. The Gcode defines the actions that the 3D printer will perform to print the object, including the movements and temperature of the printing head and bed, the rate of extrusion and retraction of the filament, and the printer's speed. At this stage, printing parameters directly affecting the object's structural integrity and aesthetics are defined. 
Critical parameters are layer height and width, the percentage and pattern of the infill, and the inclusion of support materials and rafts. Besides this, there are a number of options you can explore to optimize printing speed, material consumption and print quality. Despite this complexity, most slicing software provides an interface that enables easy manipulation of the printing settings and optimized default values. Step 4 - Printing the object Finally, the g-code file is delivered to the $3 \mathrm{D}$ printer to initialise the printing job. You can either save the g-code file to an SD card or directly upload it to the printer using a free online platform called Octoprint. For this you additionally need an internet connection, a miniaturised and affordable computer called Raspberry Pi (https: //www.raspberrypi.org), and a USB cable to connect the Raspberry Pi to the 3D printer. Setting up a Raspberry $\mathrm{Pi}$ and an OctoPrint account requires a one-time additional effort but provides useful functionalities to the user, including remote control over the $3 \mathrm{D}$ printer and the ability to live-monitor and record video timelapses of the printing jobs. We suggest DietPi as a hassle-free operating system for the Raspberry Pi (see https://dietpi.com/docs/install/ for a guide on how to easily install DietPi, and https://octoprint.org for instructions to set up an Octoprint instance). Once the G-code is delivered to the $3 \mathrm{D}$ printer, the printing job can be initialized.

Step 5 - Inspection and post-processing The final object is often ready to use right after printing. Naturally, you should inspect the quality of the printing job. For complex multi-component structures a high-quality print with perfect fit has to be assured for correct and functional assembly. Also, some models contain moving parts printed in one piece (i.e., "print-in-place" models), such as bearings and hinges. These models often require that the user forces the moving parts slightly to "release" them. Moreover, particular objects require post-processing to obtain the final structure. For example, manual removal of support structures or rafts created during printing is necessary. In other cases, for practical and aesthetic reasons, the surface of the object might require a finishing treatment such as sanding and polishing or with chemical solvents such as acetone.

Step 6 - Enjoy 3D printing 3D printing is a satisfying process: it empowers its users by enabling the materialisation of ideas. As you explore the variables involved in 3D printing and their modulation results, you will become more aware of the technology's potentials and limitations, which in turn is a vector for your creativity. 3D printing should be enjoyed, and the best results are achieved through sharing and cooperation. There are several online forums are available, where users actively engage with and benefit from the maker community by sharing their designs, advice and expertise.
Biomedical research is, for example, providing great strides in terms of biocompatible materials and coatings, particularly as $3 \mathrm{D}$ printing is a prime candidate for implant manufacturing. Environmental impact is a key factor for the use of mass production in the industry. Energy consumption will likely reduce with optimisation of the printing processes, as current 3D printers are not heavily optimised in terms of energy consumption. Plastic waste management is also a matter of standardising recycling processes to take full advantage of discarded printing materials. Pollution also depends on the optimisation of the printing processes and will likely see a reduction with time. Large scale applications will follow once 3D printing processes become more efficient and cheaper, which in turn will facilitate the accessibility of the technology. Despite current limitations, 3D printing is already enabling unprecedented customisation levels, allowing researchers to design and produce tools tailored for specific needs for low prices. 3D printing in microscopy development is particularly exploiting these capabilities, as it allows rapid prototyping and design iteration that would be otherwise difficult -if not impossible- with other fabrication technologies.

Bioprinting. The scope of 3D printing is not limited to printing polymers or similar inert materials. Biomedical researchers are currently exploring the use of "bioinks" to grow tissues and even organs in vitro. Bioinks are composed of hydrogels and cells (161). These hydrogels are either natural (e.g., alginate, chitosan, and gelatin) or engineered polymers (e.g., plastics, synthetic fibres, and elastomers) that can uptake water and act as scaffolds and protective structures to support the development of cells into tissues (162). In this sense, bioinks enable the generation of artificial 3D in vitro models recapitulating important aspects of cellular systems' physiological microenvironment. Printing with bioinks involves a specific 3D printing modality called "Direct-Ink-Writing" (DIW). Similar to FDM, DIW deposits layers of material in an organised manner to compose the final object. However, DIW uses liquid materials instead of solids. The small size of the objects produced and their high-resolution details requires precise control of material extrusion at the nanoliter scale, which is done using controlled flow rates. Commercial bioprinters are generally expensive but open-source models are also available. For example, the Replistruder 4 is an open-source syringe pumpbased model that uses a combination of 3D printed parts and mass-produced linear motion components (163).

Other attempts at achieving such complexity in experimental cellular arrangements were explored but faced critical limitations. For example, organoids are organ-like structures that recapitulate several tissue characteristics but are typically hard to reproduce and limited to structural morphologies generated spontaneously. An approach to control the morphology of artificial tissues consists in seeding cells on prefabricated synthetic scaffolds. However, due to their rigid nature, these scaffolds are intransitable and their shape cannot be modulated by the cells. Also, they substantially limit the regions where multicellular interactions can occur, and the seeding technique is unable to precisely control cellu- 
lar organisation within the tissue. Thus, this approach can only replicate the complex structure and environment of a tissue to a relatively small degree. Bioprinting using bioinks overcomes these limitations by enabling high-precision reconstruction of tissue microarchitectures in a controlled and reproducible manner. For example, a technique called "coreshell bioprinting" allows coaxial printing of two bioinks and ensures a high contact area between the two phases. Taymour et al. used this approach to create a biologically-active 3D in vitro model of the liver microenvironment containing hepatocytes and fibroblasts (164).

Furthermore, bioinks have a promising therapeutic potential, namely in the field of wound healing. For example, Nulty et al. (165) incubated a fibrin-based hydrogel containing human umbilical vein endothelial cells to generate a small in vitro vascular network. This prevascularised scaffold was then implanted into mice with critically-sized bone defects, resulting in increased vascularisation and bone formation compared to mice implanted with an immature and disorganized scaffold containing the same cellular components. These implementations showcase the therapeutic potential of 3D printing and the impact that developing this technology can have in biomedical research.

The future and reach of 3D printing. The examples of 3D printing's applications provided in this review highlight how its emergence revolutionised the manufacturing landscape. Most 3D printing techniques rely on affordable equipment and materials, bringing the accessibility of automated manufacturing processes previously exclusive to big companies down to the "hobbyist" level. Furthermore, the relatively easy accessibility to scientific devices such as 3Dprinted microscopes promotes "Citizen Science" by providing data collection tools to users who would otherwise be unable to purchase them. Thus, the manufacturing process becomes possible and cost-efficient for small- to large-scaled private projects. This autonomy precludes the need for outsourcing and the dependency on proprietary formulations or devices, which are often detrimental to project budgets and substantial limiting factors. Notably, the affordability of 3D printing technologies enables low-income countries to benefit from its advantages and the devices that it can produce. The freedom to design 3D models using open-source CAD and slicing software allows customization, facilitates prototyping, and enables sharing the designs between researchers. Furthermore, the different printing technologies and materials available, coupled with the control over several printing settings/parameters, enable users to manage crucial factors influencing their models' applicability, such as structural integrity and detail.

The establishment of 3D printing in an increasing number of instances, and the urge to benefit from its advantages, result in the continuous exposure of its current limitations. In turn, this generates a driving force for its development based on necessity. Thus, the overall advantages and limitations of $3 \mathrm{D}$ printing are expected to improve substantially in the future. Also, the relevance of the current 3D printing applications and the need to overcome their limitations make the development of 3D printing technology its own high-impact research field. In this sense, the field of microscopy is expected to continue to benefit from developments in 3D printing technologies. The development of new materials can improve current applications' success, for example, by providing biocompatible materials for sample manipulation. However, this requires the collaboration of material scientists and microscopy researchers. Furthermore, 3D printing is an ideal platform to produce compliant mechanisms, which can be used to improve current mechanical designs underlying the function of specific microscope parts. In particular, the high degree of movement precision and mechanism miniaturisation that can be achieved with 3D-printed compliant mechanisms make them a promising venue for developments in stage and sample micromovement applications.

\section{SUPPORTING INFORMATION}

Movie S1:

3D printed microscope projects (https://youtu.be/XAw4vqzV3J8)

Movie S2:

3D printed sample manipulation projects (https://youtu.be/p5-IHAZNVA8)

Movie S3:

"How to" guide for 3D printing (https://youtu.be/4jCWi2ClHyg)

Supplementary Database: 3D printing database for microscopy (https://doi.org/10. 6084/m9.figshare.14579439)

\section{ACKNOWLEDGEMENTS}

This work was supported by the Gulbenkian Foundation (M.D., H.H., A.M., R.H.) and received funding from the European Research Council (ERC) under the European Union's Horizon 2020 research and innovation programme (grant agreement No. [101001332]) (R.H.), the European Molecular Biology Organization (EMBO) Installation Grant (EMBO-2020-IG-4734) (R.H.) and the Wellcome Trust (203276/Z/16/Z) (R.H.). A.M. would like to acknowledge support from the Integrative Biology and Biomedicine PhD programme from Instituto Gulbenkian de Ciência.

\section{EXTENDED AUTHOR INFORMATION}

- Mario Del Rosario: (D0000-0002-0430-1463; ymariod

- Hannah S. Heil: (D)0000-0003-4279-7022; yannah_SuperRes

- Afonso Mendes: (D)0000-0001-7324-555X; yafonsomendes92

- Vittorio Saggiomo: (D0000-0001-7196-602X; VV_Saggiomo

- Ricardo Henriques: (1)0000-0002-2043-5234; YHenriquesLab

\section{AUTHOR CONTRIBUTIONS}

M.D., H.S.H. and A.M. contributed equally to the preparation of the manuscript, writing the document, preparing figures, tables, and movies. All authors planned structure, advised in the preparation and edited content.

\section{COMPETING FINANCIAL INTERESTS}

The authors declare no competing financial interests.

\section{Bibliography}

1. Andre Maia Chagas, Lucia L. Prieto-Godino, Aristides B. Arrenberg, and Tom Baden. The $€ 100$ lab: A 3D-printable open-source platform for fluorescence microscopy, optogenetics, and accurate temperature control during behaviour of zebrafish, Drosophila, and Caenorhabditis elegans. PLOS Biology, 15(7):e2002702, July 2017. doi: 10.1371/journal. pbio.2002702.

2. Julian Stirling, Valerian L. Sanga, Paul T. Nyakyi, Grace A. Mwakajinga, Joel T. Collins, Kaspar Bumke, Joe Knapper, Qingxin Meng, Samuel McDermott, and Richard Bowman. The OpenFlexure Project. The technical challenges of Co-Developing a microscope in the UK and Tanzania. In 2020 IEEE Global Humanitarian Technology Conference (GHTC), pages 1-4, October 2020. doi: 10.1109/GHTC46280.2020.9342860.

3. Joel T. Collins, Joe Knapper, Julian Stirling, Joram Mduda, Catherine Mkindi, Valeriana Mayagaya, Grace A. Mwakajinga, Paul T. Nyakyi, Valerian L. Sanga, Dave Carbery, Leah White, Sara Dale, Zhen Jieh Lim, Jeremy J. Baumberg, Pietro Cicuta, Samuel McDermott, Boyko Vodenicharski, and Richard Bowman. Robotic microscopy for everyone: the OpenFlexure microscope. Biomedical Optics Express, 11(5):2447-2460, May 2020. doi: 10.1364/BOE.385729.

4. Ian M. Dobbie, Emma King, Richard M. Parton, Peter M. Carlton, John W. Sedat, Jason R. Swedlow, and llan Davis. OMX: A New Platform for Multimodal, Multichannel Wide-Field Imaging. Cold Spring Harbor Protocols, 2011(8):pdb.top121, August 2011. doi: 10.1101/ pdb.top121.

5. Jürgen Sturm, Erik Bylow, Fredrik Kahl, and Daniel Cremers. CopyMe3D: Scanning and Printing Persons in 3D. In Joachim Weickert, Matthias Hein, and Bernt Schiele, editors, Pattern Recognition, Lecture Notes in Computer Science, pages 405-414, Berlin, Heidelberg, 2013. Springer. doi: 10.1007/978-3-642-40602-7_43. 
6. Jolien Van Der Putten, Alex Van Olmen, Marijke Aerts, Emiel Ascione, Joeri Beneens, Jan Blaakmeer, Geert De Schutter, and Kim Van Tittelboom. 3D Concrete Printing on Site: A Novel Way of Building Houses? In Freek P. Bos, Sandra S. Lucas, Rob J.M. Wolfs, and Theo A.M. Salet, editors, Second RILEM International Conference on Concrete and Dig ital Fabrication, RILEM Bookseries, pages 712-719, Cham, 2020. Springer International Publishing. doi: 10.1007/978-3-030-49916-7_71.

7. Tuan D. Ngo, Alireza Kashani, Gabriele Imbalzano, Kate T. Q. Nguyen, and David Hui. Additive manufacturing (3D printing): A review of materials, methods, applications and challenges. Composites Part B: Engineering, 143:172-196, June 2018. doi: 10.1016/j. compositesb.2018.02.012

8. Vojislav Petrovic, Juan Vicente Haro Gonzalez, Olga Jordá Ferrando, Javier Delgado Gordillo, Jose Ramón Blasco Puchades, and Luis Portolés Griñan. Additive layered manufacturing: sectors of industrial application shown through case studies. International Journal of Production Research, 49(4):1061-1079, February 2011. doi: 10.1080/ 00207540903479786.

9. Samuel Clark Ligon, Robert Liska, Jürgen Stampfl, Matthias Gurr, and Rolf Mülhaupt. Polymers for 3D Printing and Customized Additive Manufacturing. Chemical Reviews, 117 (15):10212-10290, August 2017. doi: 10.1021/acs.chemrev.7b00074.

10. N. Shahrubudin, T. C. Lee, and R. Ramlan. An Overview on 3D Printing Technology: Technological, Materials, and Applications. Procedia Manufacturing, 35:1286-1296, January 2019. doi: 10.1016/j.promfg.2019.06.089.

11. Antoni Van Leeuwenhoek. Observations, communicated to the publisher by Mr. Antony van Leewenhoeck, in a dutch letter of the 9th Octob. 1676. here English'd: concerning little animals by him observed in rain-well-sea- and snow water; as also in water wherein pepper had lain infused. Philosophical Transactions of the Royal Society of London, 12 (133):821-831, March 1677. doi: 10.1098/rstl.1677.0003.

12. J. Vangindertael, R. Camacho, W. Sempels, H. Mizuno, P. Dedecker, and K. P. F. Janssen. An introduction to optical super-resolution microscopy for the adventurous biologist. Methods and Applications in Fluorescence, 6(2):022003, March 2018. doi: 10.1088/2050-6120/aaae0c.

13. Koen J. A. Martens, Sam P. B. van Beljouw, Simon van der Els, Jochem N. A. Vink, Sander Baas, George A. Vogelaar, Stan J. J. Brouns, Peter van Baarlen, Michiel Kleerebezem, and Johannes Hohlbein. Visualisation of dCas 9 target search in vivo using an open-microscopy framework. Nature Communications, 10(1):3552, August 2019. doi: 10.1038/s41467-019-11514-0.

14. Benedict Diederich, René Lachmann, Swen Carlstedt, Barbora Marsikova, Haoran Wang, Xavier Uwurukundo, Alexander S. Mosig, and Rainer Heintzmann. A versatile and customizable low-cost 3D-printed open standard for microscopic imaging. Nature Communications, 11(1):5979, November 2020. doi: 10.1038/s41467-020-19447-9.

15. James W. P. Brown, Arnaud Bauer, Mark E. Polinkovsky, Akshay Bhumkar, Dominic J. B. Hunter, Katharina Gaus, Emma Sierecki, and Yann Gambin. Single-molecule detection on a portable 3D-printed microscope. Nature Communications, 10(1):5662, December 2019. doi: 10.1038/s41467-019-13617-0.

16. Stephan Amann, Max von Witzleben, and Stefan Breuer. 3D-printable portable opensource platform for low-cost lens-less holographic cellular imaging. Scientific Reports, 9 (1):11260, August 2019. doi: 10.1038/s41598-019-47689-1.

17. Samuel B. Tristan-Landin, Alan M. Gonzalez-Suarez, Rocio J. Jimenez-Valdes, and Jose L. Garcia-Cordero. Facile assembly of an affordable miniature multicolor fluorescence microscope made of 3D-printed parts enables detection of single cells. PLOS ONE, 14(10):e0215114, October 2019. doi: 10.1371/journal.pone.0215114.

18. Peter G. Pitrone, Johannes Schindelin, Luke Stuyvenberg, Stephan Preibisch, Michael Weber, Kevin W. Eliceiri, Jan Huisken, and Pavel Tomancak. OpenSPIM: an open-access light-sheet microscopy platform. Nature Methods, 10(7):598-599, July 2013. doi: 10.1038/ nmeth.2507.

19. Nemanja Vukašinović, Yaowei Wang, Isabelle Vanhoutte, Matyáš Fendrych, Boyu Guo, Miroslav Kvasnica, Petra Jiroutová, Jana Oklestkova, Miroslav Strnad, and Eugenia Russinova. Local brassinosteroid biosynthesis enables optimal root growth. bioRxiv, page 2020.11.23.391474, November 2020. doi: 10.1101/2020.11.23.391474

20. Philip R. Nicovich, James Walsh, Till Böcking, and Katharina Gaus. NicoLase-An opensource diode laser combiner, fiber launch, and sequencing controller for fluorescence microscopy. PLOS ONE, 12(3):e0173879, March 2017. doi: 10.1371/journal.pone.0173879.

21. Florian Fäßler, Bettina Zens, Robert Hauschild, and Florian K. M. Schur. 3D printed cell culture grid holders for improved cellular specimen preparation in cryo-electron microscopy. Journal of Structural Biology, 212(3):107633, December 2020. doi: 10.1016/j. jsb.2020.107633.

22. George O. T. Merces, Conor Kennedy, Blanca Lenoci, Emmanuel G. Reynaud, Niamh Burke, and Mark Pickering. The incubot: A 3D printer-based microscope for long-term live cell imaging within a tissue culture incubator. HardwareX, 9:e00189, April 2021. doi: 10.1016/j.ohx.2021.e00189.

23. Daniel M. Gruber, Tynan Perez, Bege Q. Layug, Margaret Ohama, Lydia Tran, Luis Angel Flores Rojas, A. Xavier Garcia, Gang-yu Liu, and William J. W. Miller. ThreeDimensional Printing of a Model Atomic Force Microscope to Measure Force-Distance Profiles. Journal of Chemical Education, 97(3):845-849, March 2020. doi: 10.1021/acs. jchemed.9b01099.

24. Pedro Almada, Pedro M. Pereira, Siân Culley, Ghislaine Caillol, Fanny Boroni-Rueda, Christina L. Dix, Guillaume Charras, Buzz Baum, Romain F. Laine, Christophe Leterrier, and Ricardo Henriques. Automating multimodal microscopy with NanoJ-Fluidics. Nature Communications, 10(1):1223, March 2019. doi: 10.1038/s41467-019-09231-9.

25. A. Sina Booeshaghi, Eduardo da Veiga Beltrame, Dylan Bannon, Jase Gehring, and Lior Pachter. Principles of open source bioinstrumentation applied to the poseidon syringe pump system. Scientific Reports, 9(1):12385, August 2019. doi: 10.1038/ s41598-019-48815-9.

26. Luis José Salazar-Serrano, Juan P. Torres, and Alejandra Valencia. A 3D Printed Toolbox for Opto-Mechanical Components. PLOS ONE, 12(1):e0169832, January 2017. doi: 10. 1371/journal.pone.0169832.

27. James P. Sharkey, Darryl C. W. Foo, Alexandre Kabla, Jeremy J. Baumberg, and
Richard W. Bowman. A one-piece 3D printed flexure translation stage for open-source microscopy. Review of Scientific Instruments, 87(2):025104, February 2016. doi: 10.1063/1.4941068

28. liro Hietanen, Ismo T. S. Heikkinen, Hele Savin, and Joshua M. Pearce. Approaches to open source 3-D printable probe positioners and micromanipulators for probe stations. HardwareX, 4:e00042, October 2018. doi: 10.1016/j.ohx.2018.e00042.

29. Cynthia Wong, Michal E. Pawlowski, Alessandra Forcucci, Catherine E. Majors, Rebecca Richards-Kortum, and Tomasz S. Tkaczyk. Development of a universal, tunable, miniature fluorescence microscope for use at the point of care. Biomedical Optics Express, 9(3): 1041-1056, March 2018. doi: 10.1364/BOE.9.001041.

30. Reut Orange-Kedem, Elias Nehme, Lucien E Weiss, Boris Ferdman, Onit Alalouf, Nadav Opatovski, and Yoav Shechtman. 3d printable diffractive optical elements by liquid immersion. Nature Communications, 12(1):3067, 2021. doi: 10.1038/s41467-021-23279-6.

31. Benedict Diederich, Patrick Then, Alexander Jügler, Ronny Förster, and Rainer Heintz mann. cellSTORM-Cost-effective super-resolution on a cellphone using dSTORM. PLOS ONE, 14(1):e0209827, January 2019. doi: 10.1371/journal.pone.0209827.

32. Siyuan Dong, Kaikai Guo, Pariksheet Nanda, Radhika Shiradkar, and Guoan Zheng. FPscope: a field-portable high-resolution microscope using a cellphone lens. Biomedical Optics Express, 5(10):3305-3310, October 2014. doi: 10.1364/BOE.5.003305.

33. Luís Rosado, João Oliveira, Maria João M. Vasconcelos, José M. Correia da Costa, Dirk Elias, and Jaime S. Cardoso. $\mu$ SmartScope: 3D-printed Smartphone Microscope with Motorized Automated Stage. pages 38-48, April 2021. doi: 10.5220/0006155800380048.

34. Hojeong Yu, Yafang Tan, and Brian T. Cunningham. Smartphone Fluorescence Spectroscopy. Analytical Chemistry, 86(17):8805-8813, September 2014. doi: 10.1021/ ac502080t.

35. Scott V. Angus, Soohee Cho, Dustin K. Harshman, Jae-Young Song, and Jeong-Yeol Yoon. A portable, shock-proof, surface-heated droplet PCR system for Escherichia coli detection. Biosensors \& Bioelectronics, 74:360-368, December 2015. doi: 10.1016/j.bios.2015.06. 026.

36. Siddharth Rawat, Satoru Komatsu, Adam Markman, Arun Anand, and Bahram Javidi. Compact and field-portable 3D printed shearing digital holographic microscope for automated cell identification. Applied Optics, 56(9):D127-D133, March 2017. doi: 10.1364/AO.56.00D127.

37. Alessandra Forcucci, Michal E. Pawlowski, Catherine Majors, Rebecca Richards-Kortum, and Tomasz S. Tkaczyk. All-plastic, miniature, digital fluorescence microscope for three part white blood cell differential measurements at the point of care. Biomedical Optics Express, 6(11):4433-4446, November 2015. doi: 10.1364/BOE.6.004433.

38. Vilhelm Müller, José M. Sousa, Hatice Ceylan Koydemir, Muhammed Veli, Derek Tseng, Laura Cerqueira, Aydogan Ozcan, Nuno F. Azevedo, and Fredrik Westerlund. Identification of pathogenic bacteria in complex samples using a smartphone based fluorescence microscope. RSC Advances, 8(64):36493-36502, October 2018. doi: 10.1039/C8RA06473C

39. Temitope E. Agbana, Jan-Carel Diehl, Fiona van Pul, Shahid M. Khan, Vsevolod Patlan, Michel Verhaegen, and Gleb Vdovin. Imaging \& identification of malaria parasites using cellphone microscope with a ball lens. PLOS ONE, 13(10):e0205020, October 2018. doi: 10.1371/journal.pone.0205020.

40. Haoran Wang, René Lachmann, Barbora Marsikova, Rainer Heintzmann, and Benedict Diederich. UCsim2: 2D Structured Illumination Microscopy using UC2. bioRxiv, January 2021. doi: 10.1101/2021.01.08.425840.

41. Mihails Delmans and Jim Haseloff. $\mu$ Cube: A Framework for 3D Printable Optomechanics. Journal of Open Hardware, 2(1):2, May 2018. doi: 10.5334/joh.8.

42. Richard W. Bowman, Boyko Vodenicharski, Joel T. Collins, and Julian Stirling. Flat-Field and Colour Correction for the Raspberry Pi Camera Module. Journal of Open Hardware, 4(1):1, April 2020. doi: 10.5334/joh.20.

43. Joel T. Collins, Joe Knapper, Julian Stirling, Samuel McDermott, and Richard Bowman. Modern Microscopy with the Web of Things: The OpenFlexure Microscope Software Stack. arXiv:2101.00933 [physics], January 2021. arXiv: 2101.00933.

44. Nils Gustafsson, Siân Culley, George Ashdown, Dylan M. Owen, Pedro Matos Pereira, and Ricardo Henriques. Fast live-cell conventional fluorophore nanoscopy with ImageJ through super-resolution radial fluctuations. Nature Communications, 7(1):12471, August 2016. doi: $10.1038 /$ ncomms 12471 .

45. Stephen D. Grant, Gemma S. Cairns, Jordan Wistuba, and Brian R. Patton. Adapting the 3D-printed Openflexure microscope enables computational super-resolution imaging F1000Research, 8:2003, November 2019. doi: 10.12688/f1000research.21294.1.

46. Benedict Diederich, Øystein Helle, Patrick Then, Pablo Carravilla, Kay Oliver Schink, Franziska Hornung, Stefanie Deinhardt-Emmer, Christian Eggeling, Balpreet Singh Ahluwalia, and Rainer Heintzmann. Nanoscopy on the Chea(i)p. bioRxiv, page 2020.09.04.283085, September 2020. doi: 10.1101/2020.09.04.283085.

47. Anna Archetti, Evgenii Glushkov, Christian Sieben, Anton Stroganov, Aleksandra Radenovic, and Suliana Manley. Waveguide-PAINT offers an open platform for large field-ofview super-resolution imaging. Nature Communications, 10(1):1267, March 2019. doi: 10.1038/s41467-019-09247-1.

48. Wiebke Jahr, Benjamin Schmid, Michael Weber, and Jan Huisken. eduSPIM: Light Sheet Microscopy in the Museum. PLOS ONE, 11(8):e0161402, aug 2016. doi: 10.1371/journal. pone.0161402.

49. Johannes Girstmair, Anne Zakrzewski, François Lapraz, Mette Handberg-Thorsager, Pavel Tomancak, Peter Gabriel Pitrone, Fraser Simpson, and Maximilian J. Telford. Lightsheet microscopy for everyone? Experience of building an OpenSPIM to study flatworm development. BMC Developmental Biology, 16(1):22, December 2016. doi: 10.1186/s12861-016-0122-0.

50. Zachary F. Phillips, Michael V. D’Ambrosio, Lei Tian, Jared J. Rulison, Hurshal S. Patel, Nitin Sadras, Aditya V. Gande, Neil A. Switz, Daniel A. Fletcher, and Laura Waller. MultiContrast Imaging and Digital Refocusing on a Mobile Microscope with a Domed LED Array. PLOS ONE, 10(5):e0124938, May 2015. doi: 10.1371/journal.pone.0124938.

51. Chris Stewart and John Giannini. Inexpensive, Open Source Epifluorescence Microscopes. Journal of Chemical Education, 93(7):1310-1315, jul 2016. doi: 10.1021/acs. jchemed.5b00984. 
52. Matthew Wincott, Andrew Jefferson, Ian M. Dobbie, Martin J. Booth, Ilan Davis, and Richard M. Parton. Democratising "Microscopi": a 3D printed automated XYZT fluorescence imaging system for teaching, outreach and fieldwork. Wellcome Open Research, 6: 63, March 2021. doi: 10.12688/wellcomeopenres.16536.1.

53. Sally A. N. Gowers, Vincenzo F. Curto, Carlo A. Seneci, Chu Wang, Salzitsa Anastasova, Pankaj Vadgama, Guang-Zhong Yang, and Martyn G. Boutelle. 3D Printed Microfluidic Device with Integrated Biosensors for Online Analysis of Subcutaneous Human Microdialysate. Analytical Chemistry, 87(15):7763-7770, August 2015. doi: 10.1021/acs. analchem.5b01353.

54. Kyoung G. Lee, Kyun Joo Park, Seunghwan Seok, Sujeong Shin, Do Hyun Kim, Jung Youn Park, Yun Seok Heo, Seok Jae Lee, and Tae Jae Lee. 3D printed modules for integrated microfluidic devices. RSC Advances, 4(62):32876-32880, July 2014. doi: 10.1039/C4RA05072J.

55. R. D. Sochol, E. Sweet, C. C. Glick, S. Venkatesh, A. Avetisyan, K. F. Ekman, A. Raulinaitis, A. Tsai, A. Wienkers, K. Korner, K. Hanson, A. Long, B. J. Hightower, G. Slatton, D. C. Burnett, T. L. Massey, K. Iwai, L. P. Lee, K. S. J. Pister, and L. Lin. 3D printed microfluidic circuitry via multijet-based additive manufacturing. Lab on a Chip, 16(4):668678, February 2016. doi: 10.1039/C5LC01389E.

56. Yuan-Sheng Lee, Nirveek Bhattacharjee, and Albert Folch. 3D-printed Quake-style microvalves and micropumps. Lab on a Chip, 18(8):1207-1214, April 2018. doi: 10.1039/ C8LC00001H.

57. Michael R. Behrens, Haley C. Fuller, Emily R. Swist, Jingwen Wu, Md Mydul Islam, Zhicheng Long, Warren C. Ruder, and Robert Steward. Open-source, 3D-printed Peristaltic Pumps for Small Volume Point-of-Care Liquid Handling. Scientific Reports, 10(1): 1543, January 2020. doi: 10.1038/s41598-020-58246-6.

58. Sidra Waheed, Joan M. Cabot, Niall P. Macdonald, Trevor Lewis, Rosanne M. Guijt, Brett Paull, and Michael C. Breadmore. 3D printed microfluidic devices: enablers and barriers. Lab on a Chip, 16(11):1993-2013, May 2016. doi: 10.1039/C6LC00284F.

59. Muhd Nazrul Hisham Zainal Alam, Faruque Hossain, Alexander Vale, and Abbas Kouzani. Design and fabrication of a 3D printed miniature pump for integrated microfluidic applications. International Journal of Precision Engineering and Manufacturing, 18(9):1287-1296, September 2017. doi: 10.1007/s12541-017-0152-y.

60. Anthony K. Au, Nirveek Bhattacharjee, Lisa F. Horowitz, Tim C. Chang, and Albert Folch. 3D-printed microfluidic automation. Lab on a Chip, 15(8):1934-1941, March 2015. doi: 10.1039/C5LC00126A.

61. Alexander Jönsson, Arianna Toppi, and Martin Dufva. The FAST Pump, a low-cost, easy to fabricate, SLA-3D-printed peristaltic pump for multi-channel systems in any lab. HardwareX, 8:e00115, October 2020. doi: 10.1016/j.ohx.2020.e00115.

62. Hua Gong, Adam T. Woolley, and Gregory P. Nordin. High density 3D printed microfluidic valves, pumps, and multiplexers. Lab on a Chip, 16(13):2450-2458, June 2016. doi: 10.1039/C6LC00565A.

63. C. Sun, N. Fang, D. M. Wu, and X. Zhang. Projection micro-stereolithography using digital micro-mirror dynamic mask. Sensors and Actuators A: Physical, 121(1):113-120, May 2005. doi: 10.1016/j.sna.2004.12.011.

64. Kati Piironen, Markus Haapala, Virpi Talman, Päivi Järvinen, and Tiina Sikanen. Cell adhesion and proliferation on common 3D printing materials used in stereolithography of microfluidic devices. Lab on a Chip, 20(13):2372-2382, June 2020. doi: 10.1039/ D0LC00114G.

65. Carlos Ruiz, Karteek Kadimisetty, Kun Yin, Michael G. Mauk, Hui Zhao, and Changchun Liu. Fabrication of Hard-Soft Microfluidic Devices Using Hybrid 3D Printing. Micromachines, 11(6):567, June 2020. doi: 10.3390/mi11060567.

66. Gregor Weisgrab, Aleksandr Ovsianikov, and Pedro F. Costa. Functional 3D Printing fo Microfluidic Chips. Advanced Materials Technologies, 4(10):1900275, 2019. doi: https: //doi.org/10.1002/admt.201900275.

67. Hua Gong, Bryce P. Bickham, Adam T. Woolley, and Gregory P. Nordin. Custom 3D printer and resin for $18 \mu \mathrm{m} \times 20 \mu \mathrm{m}$ microfluidic flow channels. Lab on a Chip, 17(17):2899-2909, August 2017. doi: 10.1039/C7LC00644F.

68. Tobias Dahlberg, Tim Stangner, Hanqing Zhang, Krister Wiklund, Petter Lundberg, Ludvig Edman, and Magnus Andersson. 3D printed water-soluble scaffolds for rapid production of PDMS micro-fluidic flow chambers. Scientific Reports, 8(1):3372, February 2018. doi: 10.1038/s41598-018-21638-w

69. Vittorio Saggiomo and Aldrik H Velders. Simple 3d printed scaffold-removal method for the fabrication of intricate microfluidic devices. Advanced science, 2(9):1500125, 2015. doi: 10.1002/advs.201500125.

70. Stan BJ Willems, Jaccoline Zegers, Anton Bunschoten, R Martijn Wagterveld, Fijs WB Van Leeuwen, Aldrik H Velders, and Vittorio Saggiomo. Covalent monolayer patterns in microfluidics by plasma etching open technology-complot. Analyst, 145(5):1629-1635, 2020. doi: 10.1039/C9AN02407G.

71. Sajad Razavi Bazaz, Omid Rouhi, Mohammad Amin Raoufi, Fatemeh Ejeian, Mohsen Asadnia, Dayong Jin, and Majid Ebrahimi Warkiani. 3D Printing of Inertial Microfluidic Devices. Scientific Reports, 10(1):5929, April 2020. doi: 10.1038/s41598-020-62569-9.

72. Alessandro Iuliano, Erik van der Wal, Claudine W. B. Ruijmbeek, Stijn L. M. in 't Groen, W. W. M. Pim Pijnappel, Jessica C. de Greef, and Vittorio Saggiomo. Coupling 3D Printing and Novel Replica Molding for In House Fabrication of Skeletal Muscle Tissue Engineering Devices. Advanced Materials Technologies, 5(9):2000344, 2020. doi: https://doi.org/10. 1002/admt.202000344.

73. Dagmar Chudobova, Kristyna Cihalova, Sylvie Skalickova, Jan Zitka, Miguel Angel Merlos Rodrigo, Vedran Milosavljevic, David Hynek, Pavel Kopel, Radek Vesely, Vojtech Adam, and Rene Kizek. 3D-printed chip for detection of methicillin-resistant Staphylococcus aureus labeled with gold nanoparticles. ELECTROPHORESIS, 36(3):457-466, 2015. doi: https://doi.org/10.1002/elps.201400321.

74. Yangfan Chen, Ho Nam Chan, Sean A. Michael, Yusheng Shen, Yin Chen, Qian Tian, Lu Huang, and Hongkai Wu. A microfluidic circulatory system integrated with capillaryassisted pressure sensors. Lab on a Chip, 17(4):653-662, February 2017. doi: 10.1039/ C6LC01427E.

75. Sara Hinds, Weining Bian, Robert G. Dennis, and Nenad Bursac. The role of extracellular matrix composition in structure and function of bioengineered skeletal muscle. Biomaterials, 32(14):3575-3583, May 2011. doi: 10.1016/j.biomaterials.2011.01.062.

76. Philip J. Lee, Paul J. Hung, and Luke P. Lee. An artificial liver sinusoid with a microfluidic endothelial-like barrier for primary hepatocyte culture. Biotechnology and Bioengineering, 97(5):1340-1346, 2007. doi: https://doi.org/10.1002/bit.21360.

77. Siming Lu, Fabio Cuzzucoli, Jing Jiang, Li-Guo Liang, Yimin Wang, Mengqi Kong, Xin Zhao, Wenguo Cui, Jun Li, and ShuQi Wang. Development of a biomimetic liver tumoron-a-chip model based on decellularized liver matrix for toxicity testing. Lab on a Chip, 18 (22):3379-3392, November 2018. doi: 10.1039/C8LC00852C

78. Young Bok (Abraham) Kang, Temitope R. Sodunke, Jason Lamontagne, Joseph Cirillo, Caroline Rajiv, Michael J. Bouchard, and Moses Noh. Liver sinusoid on a chip: Long-term layered co-culture of primary rat hepatocytes and endothelial cells in microfluidic platforms. Biotechnology and Bioengineering, 112(12):2571-2582, 2015. doi: https://doi.org/10.1002/ bit.25659.

79. Qing Zhou, Dipali Patel, Timothy Kwa, Amranul Haque, Zimple Matharu, Gulnaz Stybayeva, Yandong Gao, Anna Mae Diehl, and Alexander Revzin. Liver injury-on-a-chip: microfluidic co-cultures with integrated biosensors for monitoring liver cell signaling during injury. Lab on a Chip, 15(23):4467-4478, November 2015. doi: 10.1039/C5LC00874C.

80. Ikram Khan, Anil Prabhakar, Chloe Delepine, Hayley Tsang, Vincent Pham, and Mriganka Sur. A low-cost 3D printed microfluidic bioreactor and imaging chamber for live-organoid imaging. Biomicrofluidics, 15(2):024105, March 2021. doi: 10.1063/5.0041027.

81. Dongeun Huh, Benjamin D. Matthews, Akiko Mammoto, Martín Montoya-Zavala, Hong Yuan Hsin, and Donald E. Ingber. Reconstituting Organ-Level Lung Functions on Chip. Science, 328(5986):1662-1668, June 2010. doi: 10.1126/science.1188302.

82. Dongeun Huh, Daniel C. Leslie, Benjamin D. Matthews, Jacob P. Fraser, Samuel Jurek, Geraldine A. Hamilton, Kevin S. Thorneloe, Michael Allen McAlexander, and Donald E. Ingber. A Human Disease Model of Drug Toxicity-Induced Pulmonary Edema in a Lung-ona-Chip Microdevice. Science Translational Medicine, 4(159):159ra147-159ra147, November 2012. doi: 10.1126/scitranslmed.3004249.

83. Kyung-Jin Jang, Ali Poyan Mehr, Geraldine A. Hamilton, Lori A. McPartlin, Seyoon Chung, Kahp-Yang Suh, and Donald E. Ingber. Human kidney proximal tubule-on-a-chip for drug transport and nephrotoxicity assessment. Integrative Biology, 5(9):1119-1129, September 2013. doi: $10.1039 / \mathrm{c} 3 i b 40049 \mathrm{~b}$

84. Frans Schutgens, Maarten B. Rookmaaker, Thanasis Margaritis, Anne Rios, Carola Ammerlaan, Jitske Jansen, Linda Gijzen, Marianne Vormann, Annelotte Vonk, Marco Viveen, Fjodor Yousef Yengej, Sepide Derakhshan, Karin M. de Winter-de Groot, Benedetta Artegiani, Ruben van Boxtel, Edwin Cuppen, Antoni P. A. Hendrickx, Marry M. van den Heuvel-Eibrink, Ellen Heitzer, Henriette Lanz, Jeffrey Beekman, Jean-Luc Murk, Rosalinde Masereeuw, Frank Holstege, Jarno Drost, Marianne C. Verhaar, and Hans Clevers. Tubuloids derived from human adult kidney and urine for personalized disease modeling. Nature Biotechnology, 37(3):303-313, March 2019. doi: 10.1038/s41587-019-0048-8.

85. Hyun Jung Kim, Dongeun Huh, Geraldine Hamilton, and Donald E. Ingber. Human gut-ona-chip inhabited by microbial flora that experiences intestinal peristalsis-like motions and flow. Lab on a Chip, 12(12):2165-2174, May 2012. doi: 10.1039/C2LC40074J.

86. Sasan Jalili-Firoozinezhad, Francesca S. Gazzaniga, Elizabeth L. Calamari, Diogo M. Camacho, Cicely W. Fadel, Amir Bein, Ben Swenor, Bret Nestor, Michael J. Cronce, Alessio Tovaglieri, Oren Levy, Katherine E. Gregory, David T. Breault, Joaquim M. S. Cabral, Dennis L. Kasper, Richard Novak, and Donald E. Ingber. A complex human gut microbiome cultured in an anaerobic intestine-on-a-chip. Nature Biomedical Engineering, 3(7):520531, July 2019. doi: 10.1038/s41551-019-0397-0.

87. Xi Zhang, Tianxing Wang, Ping Wang, and Ning Hu. High-Throughput Assessment of Drug Cardiac Safety Using a High-Speed Impedance Detection Technology-Based Hearton-a-Chip. Micromachines, 7(7):122, July 2016. doi: 10.3390/mi7070122.

88. Evangeline Tzatzalos, Oscar J. Abilez, Praveen Shukla, and Joseph C. Wu. Engineered heart tissues and induced pluripotent stem cells: Macro- and microstructures for disease modeling, drug screening, and translational studies. Advanced Drug Delivery Reviews, 96: 234-244, January 2016. doi: 10.1016/j.addr.2015.09.010.

89. Cacie Hart, Charles M. Didier, Frank Sommerhage, and Swaminathan Rajaraman. Biocompatibility of Blank, Post-Processed and Coated 3D Printed Resin Structures with Electrogenic Cells. Biosensors, 10(11):152, November 2020. doi: 10.3390/bios10110152.

90. C. Morrison, R. Macnair, C. MacDonald, A. Wykman, I. Goldie, and M. H. Grant. In vitro biocompatibility testing of polymers for orthopaedic implants using cultured fibroblasts and osteoblasts. Biomaterials, 16(13):987-992, September 1995. doi: 10.1016/0142-9612(95) 94906-2.

91. Abid Haleem and Mohd Javaid. Polyether ether ketone (PEEK) and its 3D printed implants applications in medical field: An overview. Clinical Epidemiology and Global Health, 7(4): 571-577, December 2019. doi: 10.1016/j.cegh.2019.01.003.

92. Kevin Alessandri, Laetitia Andrique, Maxime Feyeux, Andreas Bikfalvi, Pierre Nassoy, and Gaëlle Recher. All-in-one 3D printed microscopy chamber for multidimensional imaging, the UniverSlide. Scientific Reports, 7(1):42378, February 2017. doi: 10.1038/srep42378.

93. Daniel J. Shiwarski, Joshua W. Tashman, Amity F. Eaton, Gerard Apodaca, and Adam W. Feinberg. 3D printed biaxial stretcher compatible with live fluorescence microscopy. HardwareX, 7:e00095, April 2020. doi: 10.1016/j.ohx.2020.e00095.

94. Dominik Schneidereit, Jessica Tschernich, Oliver Friedrich, Marina Scharin-Mehlmann, and Daniel F. Gilbert. 3D-Printed Reusable Cell Culture Chamber with Integrated Electrodes for Electrical Stimulation and Parallel Microscopic Evaluation. 3D Printing and Additive Manufacturing, 5(2):115-125, June 2018. doi: 10.1089/3dp.2017.0103.

95. S. Seriani, G. Del Favero, J. Mahaffey, D. Marko, P. Gallina, C. S. Long, L. Mestroni, and O. Sbaizero. The cell-stretcher: A novel device for the mechanical stimulation of cell populations. Review of Scientific Instruments, 87(8):084301, August 2016. doi: $10.1063 / 1.4959884$

96. Nourin Alsharif, Anna Burkatovsky, Charles Lissandrello, Keith M. Jones, Alice E. White, and Keith A. Brown. Design and Realization of 3D Printed AFM Probes. Small, 14(19): 1800162, 2018. doi: https://doi.org/10.1002/smll.201800162.

97. Daniel J. Müller and Yves F. Dufrêne. Atomic force microscopy as a multifunctional molecular toolbox in nanobiotechnology. Nature Nanotechnology, 3(5):261-269, May 2008. doi: 
$10.1038 /$ nnano.2008.100

98. N. Moldovan, Keun-Ho Kim, and H.D. Espinosa. Design and fabrication of a novel microfluidic nanoprobe. Journal of Microelectromechanical Systems, 15(1):204-213, February 2006. doi: 10.1109/JMEMS.2005.863701. Conference Name: Journal of Microelectromechanical Systems

99. André Meister, Michael Gabi, Pascal Behr, Philipp Studer, János Vörös, Philippe Niedermann, Joanna Bitterli, Jérôme Polesel-Maris, Martha Liley, Harry Heinzelmann, and Tomaso Zambelli. FluidFM: Combining Atomic Force Microscopy and Nanofluidics in a Universal Liquid Delivery System for Single Cell Applications and Beyond. Nano Letters, 9(6):2501-2507, June 2009. doi: 10.1021/n1901384x

100. Robert C. L. N. Kramer, Eleonoor J. Verlinden, Livia Angeloni, Anita van den Heuvel, Lidy E. Fratila-Apachitei, Silvère M. van der Maarel, and Murali K. Ghatkesar. Multiscale 3D-printing of microfluidic AFM cantilevers. Lab on a Chip, 20(2):311-319, January 2020. doi: $10.1039 /$ C9LC00668K

101. Yopi Y. Tanoto, Juliana Anggono, lan H. Siahaan, and Wesley Budiman. The effect of orientation difference in fused deposition modeling of ABS polymer on the processing time, dimension accuracy, and strength. AIP Conference Proceedings, 1788(1):030051, January 2017. doi: 10.1063/1.4968304.

102. Miguel Fernandez-Vicente, Wilson Calle, Santiago Ferrandiz, and Andres Conejero. Effect of Infill Parameters on Tensile Mechanical Behavior in Desktop 3D Printing. 3D Printing and Additive Manufacturing, 3(3):183-192, September 2016. doi: 10.1089/3dp.2015.0036.

103. William Oropallo and Les A. Piegl. Ten challenges in $3 \mathrm{D}$ printing. Engineering with Com puters, 32(1):135-148, January 2016. doi: 10.1007/s00366-015-0407-0.

104. Charles W. Hull. Apparatus for production of three-dimensional objects by stereolithography, March 1986 . Patent US4575330A, https://patents.google.com/patent/US4575330A/en.

105. Hamad B. Olayan, Halim S. Hami, and E. D. Owen. Photochemical and Thermal Crosslink ing of Polymers. Journal of Macromolecular Science, Part C, 36(4):671-719, November 1996. doi: 10.1080/15321799608014858

106. Ali Bagheri and Jianyong Jin. Photopolymerization in 3D Printing. ACS Applied Polymer Materials, 1(4):593-611, April 2019. doi: 10.1021/acsapm. 8 b00165.

107. Lars Kool, Floris Dekker, Anton Bunschoten, Glen J Smales, Brian R Pauw, Aldrik H Velders, and Vittorio Saggiomo. Gold and silver dichroic nanocomposite in the quest for 3d printing the lycurgus cup. Beilstein journal of nanotechnology, 11(1):16-23, 2020.

108. Michael Dawoud, Iman Taha, and Samy J. Ebeid. Mechanical behaviour of ABS: An experimental study using FDM and injection moulding techniques. Journal of Manufacturing Processes, 21:39-45, January 2016. doi: 10.1016/j.jmapro.2015.11.002.

109. Martin Seidl, Jiri Safka, Jiri Bobek, Lubos Behalek, and Jiri Habr. Mechanical properties of products made of abs with respect to individuality of fdm production porcess, February 2017

110. Shilpesh R. Rajpurohit and Harshit K. Dave. Effect of process parameters on tensile strength of FDM printed PLA part. Rapid Prototyping Journal, 24(8):1317-1324, January 2018. doi: 10.1108/RPJ-06-2017-0134.

111. Shilpesh R. Rajpurohit and Harshit K. Dave. Analysis of tensile strength of a fused filament fabricated PLA part using an open-source 3D printer. The International Journal of Advanced Manufacturing Technology, 101(5):1525-1536. April 2019. doi: 10.1007/ s00170-018-3047-x

112. Nagendra G. Tanikella, Ben Wittbrodt, and Joshua M. Pearce. Tensile strength of commercial polymer materials for fused filament fabrication 3D printing. Additive Manufacturing 15:40-47, May 2017. doi: 10.1016/j.addma.2017.03.005.

113. Makara Lay, Nuur Laila Najwa Thajudin, Zuratul Ain Abdul Hamid, Arjulizan Rusli, Muhammad Khalil Abdullah, and Raa Khimi Shuib. Comparison of physical and mechanica properties of PLA, ABS and nylon 6 fabricated using fused deposition modeling and injection molding. Composites Part B: Engineering, 176:107341, November 2019. doi: 10.1016/j.compositesb.2019.107341.

114. Mohanad Idrees, Shaik Jeelani, and Vijaya Rangari. Three-Dimensional-Printed Sustainable Biochar-Recycled PET Composites. ACS Sustainable Chemistry \& Engineering, 6 (11):13940-13948, November 2018. doi: 10.1021/acssuschemeng.8b02283.

115. Sofiane Guessasma, Sofiane Belhabib, and Hedi Nouri. Printability and Tensile Performance of 3D Printed Polyethylene Terephthalate Glycol Using Fused Deposition Modelling Polymers, 11(7):1220, July 2019. doi: 10.3390/polym11071220.

116. Ksawery Szykiedans, Wojciech Credo, and Dymitr Osiński. Selected Mechanical Properties of PETG 3-D Prints. Procedia Engineering, 177:455-461, January 2017. doi: 10.1016/j.proeng.2017.02.245.

117. Matthew J. Reich, Aubrey L. Woern, Nagendra G. Tanikella, and Joshua M. Pearce. Mechanical Properties and Applications of Recycled Polycarbonate Particle Materia Extrusion-Based Additive Manufacturing. Materials, 12(10):1642, January 2019. doi: 10.3390/ma12101642.

118. Peng Geng, Ji Zhao, Wenzheng Wu, Wenli Ye, Yulei Wang, Shuobang Wang, and Shuo Zhang. Effects of extrusion speed and printing speed on the 3D printing stability of extruded PEEK filament. Journal of Manufacturing Processes, 37:266-273, January 2019. doi: 10.1016/j.jmapro.2018.11.023.

119. Shouling Ding, Bin Zou, Peng Wang, and Hongjian Ding. Effects of nozzle temperature and building orientation on mechanical properties and microstructure of PEEK and PEI printed by 3D-FDM. Polymer Testing, 78:105948, September 2019. doi: 10.1016/j.polymertesting. 2019.105948

120. Jun Wang, Bin Yang, Xiang Lin, Lei Gao, Tao Liu, Yonglai Lu, and Runguo Wang. Research of tpu materials for $3 \mathrm{~d}$ printing aiming at non-pneumatic tires by fdm method. Polymers, 11(12), 2020. doi: 10.3390/polym12112492.

121. Mostafa Nikzad, Syed H. Masood, Igor Sbarski, and Andrew M. Groth. Rheological Prop erties of a Particulate-Filled Polymeric Composite through Fused Deposition Process. Materials Science Forum, 654-656:2471-2474, 2010. doi: 10.4028/www.scientific.net/MSF. 654-656.2471.

122. Tuan Noraihan Azila Tuan Rahim, Abdul Manaf Abdullah, and Hazizan Md Akil. Recen Developments in Fused Deposition Modeling-Based 3D Printing of Polymers and Their Composites. Polymer Reviews, 59(4):589-624, October 2019. doi: 10.1080/15583724.
2019.1597883.

123. Jabran Saroia, Yanen Wang, Qinghua Wei, Mingju Lei, Xinpei Li, Ying Guo, and Kun Zhang. A review on 3D printed matrix polymer composites: its potential and future challenges. The International Journal of Advanced Manufacturing Technology, 106(5):16951721, January 2020. doi: 10.1007/s00170-019-04534-z.

124. Nanya Li, Yingguang $\mathrm{Li}$, and Shuting Liu. Rapid prototyping of continuous carbon fiber reinforced polylactic acid composites by 3D printing. Journal of Materials Processing Technology, 238:218-225, December 2016. doi: 10.1016/j.jmatprotec.2016.07.025.

125. Weihong Zhong, Fan Li, Zuoguang Zhang, Lulu Song, and Zhimin Li. Short fiber reinforced composites for fused deposition modeling. Materials Science and Engineering: A, 301(2): 125-130, March 2001. doi: 10.1016/S0921-5093(00)01810-4.

126. Sachini Wickramasinghe, Truong Do, and Phuong Tran. FDM-Based 3D Printing of Polymer and Associated Composite: A Review on Mechanical Properties, Defects and Treatments. Polymers, 12(7):1529, July 2020. doi: 10.3390/polym12071529.

127. Jim H. Lee, Robert K. Prud'homme, and Ilhan A. Aksay. Cure depth in photopolymerization: Experiments and theory. Journal of Materials Research, 16(12):3536-3544, December 2001. doi: 10.1557/JMR.2001.0485.

128. Sigrid Jauk and Robert Liska. Photoinitiators with Functional Groups, 8. Macromolec ular Rapid Communications, 26(21):1687-1692, 2005. doi: https://doi.org/10.1002/marc. 200500507.

129. Ali Bagheri, Hamidreza Arandiyan, Nik Nik M. Adnan, Cyrille Boyer, and May Lim. Controlled Direct Growth of Polymer Shell on Upconversion Nanoparticle Surface via Visible Light Regulated Polymerization. Macromolecules, 50(18):7137-7147, September 2017. doi: 10.1021/acs.macromol.7b01405.

130. Assi Al Mousawi, Patxi Garra, Michael Schmitt, Joumana Toufaily, Tayssir Hamieh, Bernadette Graff, Jean Pierre Fouassier, Frederic Dumur, and Jacques Lalevée. 3 Hydroxyflavone and N-Phenylglycine in High Performance Photoinitiating Systems for 3D Printing and Photocomposites Synthesis. Macromolecules, 51(12):4633-4641, June 2018. doi: 10.1021/acs.macromol.8b00979.

131. Marek Pagac, Jiri Hajnys, Quoc-Phu Ma, Lukas Jancar, Jan Jansa, Petr Stefek, and Jakub Mesicek. A Review of Vat Photopolymerization Technology: Materials, Applications, Challenges, and Future Trends of 3D Printing. Polymers, 13(4):598, January 2021 doi: $10.3390 /$ polym 13040598 .

132. Haoyuan Quan, Ting Zhang, Hang Xu, Shen Luo, Jun Nie, and Xiaoqun Zhu. Photo-curing 3D printing technique and its challenges. Bioactive Materials, 5(1):110-115, March 2020. doi: 10.1016/j.bioactmat.2019.12.003.

133. N. P. Macdonald, F. Zhu, C. J. Hall, J. Reboud, P. S. Crosier, E. E. Patton, D. Wlodkowic, and J. M. Cooper. Assessment of biocompatibility of 3D printed photopolymers using zebrafish embryo toxicity assays. Lab on a Chip, 16(2):291-297, January 2016. doi: 10.1039/C5LC01374G.

134. Xin Wang, Man Jiang, Zuowan Zhou, Jihua Gou, and David Hui. 3D printing of polymer matrix composites: A review and prospective. Composites Part B: Engineering, 110:442458, February 2017. doi: 10.1016/j.compositesb.2016.11.034

135. A. Le Duigou, M. Castro, R. Bevan, and N. Martin. 3D printing of wood fibre biocomposites: From mechanical to actuation functionality. Materials \& Design, 96:106-114, April 2016. doi: 10.1016/j.matdes.2016.02.018.

136. Clara Minas, Davide Carnelli, Elena Tervoort, and André R. Studart. 3D Printing of Emulsions and Foams into Hierarchical Porous Ceramics. Advanced Materials, 28(45):99939999, 2016. doi: https://doi.org/10.1002/adma.201603390.

137. Sofiane Guessasma, Sofiane Belhabib, Hedi Nouri, and Omar Ben Hassana. Anisotropic damage inferred to $3 \mathrm{D}$ printed polymers using fused deposition modelling and subject to severe compression. European Polymer Journal, 85:324-340, December 2016. doi: 10.1016/j.eurpolymj.2016.10.030.

138. Rui Zou, Yang Xia, Shiyi Liu, Ping Hu, Wenbin Hou, Qingyuan Hu, and Chunlai Shan Isotropic and anisotropic elasticity and yielding of 3D printed material. Composites Part B: Engineering, 99:506-513, August 2016. doi: 10.1016/j.compositesb.2016.06.009.

139. ISO/TC 194 Biological and clinical evaluation of medical devices. ISO 10993-1:2018: Biological evaluation of medical devices - part 1: Evaluation and testing within a risk management process, October 2018 https://www.iso.org/cms/render/live/en/sites/isoorg/contents/data/standard/06/89/68936.htm .

140. Saurabh Kumar, Chandra Mouli Pandey, Amir Hatamie, Abdolreza Simchi, Magnus Willander, and Bansi D. Malhotra. Nanomaterial-Modified Conducting Paper: Fabrication, Properties, and Emerging Biomedical Applications. Global Challenges, 3(12):1900041, 2019. doi: https://doi.org/10.1002/gch2.201900041.

141. Udayabhanu Jammalamadaka and Karthik Tappa. Recent Advances in Biomaterials for 3D Printing and Tissue Engineering. Journal of Functional Biomaterials, 9(1):22, March 2018. doi: 10.3390/jfb9010022.

142. Witold Jamróz, Joanna Szafraniec, Mateusz Kurek, and Renata Jachowicz. 3D Printing in Pharmaceutical and Medical Applications - Recent Achievements and Challenges. Pharmaceutical Research, 35(9):176, July 2018. doi: 10.1007/s11095-018-2454-x.

143. Tae-Sik Jang, Hyun-Do Jung, Houwen Matthew Pan, Win Tun Han, Shenyang Chen, and Juha Song. 3D printing of hydrogel composite systems: Recent advances in technology for tissue engineering. International Journal of Bioprinting, 4(1), January 2018. doi: 10 18063/ijb.v4i1.126.

144. Derek H. Rosenzweig, Eric Carelli, Thomas Steffen, Peter Jarzem, and Lisbet Haglund. 3D-Printed ABS and PLA Scaffolds for Cartilage and Nucleus Pulposus Tissue Regeneration. International Journal of Molecular Sciences, 16(7):15118-15135, July 2015. doi: 10.3390/ijms 160715118.

145. Paul M. van Midwoud, Arnout Janse, Marjolijn T. Merema, Geny M. M. Groothuis, and Elisabeth Verpoorte. Comparison of Biocompatibility and Adsorption Properties of Different Plastics for Advanced Microfluidic Cell and Tissue Culture Models. Analytical Chemistry, 84(9):3938-3944, May 2012. doi: 10.1021/ac300771z.

146. Yan Jiang, Yuan Liang, Hongwen Zhang, Weiwei Zhang, and Shanshan Tu. Preparation and biocompatibility of grafted functional $\beta$-cyclodextrin copolymers from the surface of PET films. Materials Science and Engineering: C, 41:1-7, August 2014. doi: 10.1016/j. msec.2014.04.031. 
147. Shirin Mesbah Oskui, Graciel Diamante, Chunyang Liao, Wei Shi, Jay Gan, Daniel Schlenk, and William H. Grover. Assessing and Reducing the Toxicity of 3D-Printed Parts. Environmental Science \& Technology Letters, 3(1):1-6, January 2016. doi: 10.1021/acs.estlett.5b00249.

148. Frank Alifui-Segbaya, Sony Varma, Graham J. Lieschke, and Roy George. Biocompatibility of Photopolymers in 3D Printing. 3D Printing and Additive Manufacturing, 4(4):185-191, December 2017. doi: 10.1089/3dp.2017.0064.

149. Gustavo González, Désirée Baruffaldi, Cinzia Martinengo, Angelo Angelini, Annalisa Chiappone, Ignazio Roppolo, Candido Fabrizio Pirri, and Francesca Frascella. Materials Testing for the Development of Biocompatible Devices through Vat-Polymerization 3D Printing. Nanomaterials (Basel, Switzerland), 10(9), September 2020. doi: 10.3390/nano10091788.

150. Yoshinori Inoue and Koji Ikuta. Detoxification of the Photocurable Polymer by Heat Treatment for Microstereolithography. Procedia CIRP, 5:115-118, January 2013. doi: 10.1016/j.procir.2013.01.023.

151. Marina Popal, Joachim Volk, Gabriele Leyhausen, and Werner Geurtsen. Cytotoxic and genotoxic potential of the type I photoinitiators BAPO and TPO on human oral keratinocytes and V79 fibroblasts. Dental Materials, 34(12):1783-1796, December 2018. doi: 10.1016/j.dental.2018.09.015.

152. Kurt Geckeler, Roland Wacker, Franz Martini, Anita Hack, and Wilhelm Aicher. Enhanced Biocompatibility for SAOS-2 Osteosarcoma Cells by Surface Coating with Hydrophobic Epoxy Resins. Cellular Physiology and Biochemistry, 13(3):155-164, 2003. doi: 10.1159/ 000071866.

153. Gert IJ. Salentijn, Pieter E. Oomen, Maciej Grajewski, and Elisabeth Verpoorte. Fused Deposition Modeling 3D Printing for (Bio)analytical Device Fabrication: Procedures, Materials, and Applications. Analytical Chemistry, 89(13):7053-7061, July 2017. doi: 10.1021/acs.analchem.7b00828.

154. David Rejeski, Fu Zhao, and Yong Huang. Research needs and recommendations on environmental implications of additive manufacturing. Additive Manufacturing, 19:21-28, January 2018. doi: 10.1016/j.addma.2017.10.019.

155. Mohammad Reza Khosravani and Tamara Reinicke. On the environmental impacts of 3D printing technology. Applied Materials Today, 20:100689, September 2020. doi: 10.1016/ j.apmt.2020.100689.

156. Tobias Kamps, Max Lutter-Guenther, Christian Seidel, Timothy Gutowski, and Gunther Reinhart. Cost- and energy-efficient manufacture of gears by laser beam melting. CIRP Journal of Manufacturing Science and Technology, 21:47-60, May 2018. doi: 10.1016/j. cirpj.2018.01.002.

157. Simon Ford and Mélanie Despeisse. Additive manufacturing and sustainability: an exploratory study of the advantages and challenges. Journal of Cleaner Production, 137: 1573-1587, November 2016. doi: 10.1016/j.jclepro.2016.04.150.

158. Kevin R. Hart, Jolie B. Frketic, and John R. Brown. Recycling meal-ready-to-eat (MRE) pouches into polymer filament for material extrusion additive manufacturing. Additive Manufacturing, 21:536-543, May 2018. doi: 10.1016/j.addma.2018.04.011.

159. S. Wojtyła, P. Klama, K. Śpiewak, and T. Baran. 3D printer as a potential source of indoor air pollution. International Journal of Environmental Science and Technology, 17(1):207218, January 2020. doi: 10.1007/s13762-019-02444-x.

160. Vincent SD Voet, Tobias Strating, Geraldine HM Schnelting, Peter Dijkstra, Martin Tietema, Jin Xu, Albert JJ Woortman, Katja Loos, Jan Jager, and Rudy Folkersma. Biobased acrylate photocurable resin formulation for stereolithography 3d printing. Acs Omega, 3(2): 1403-1408, 2018. doi: 10.1021/acsomega.7b01648.

161. Enrique Mancha Sánchez, J. Carlos Gómez-Blanco, Esther López Nieto, Javier G. Casado, Antonio Macías-García, María A. Díaz Díez, Juan Pablo Carrasco-Amador, Diego Torrejón Martín. Francisco Miguel Sánchez-Margallo, and J. Blas Pagador. Hydrogels for Bioprinting: A Systematic Review of Hydrogels Synthesis, Bioprinting Parameters, and Bioprinted Structures Behavior. Frontiers in Bioengineering and Biotechnology, 8, 2020. doi: 10.3389/fbioe.2020.00776.

162. Xinda Li, Boxun Liu, Ben Pei, Jianwei Chen, Dezhi Zhou, Jiayi Peng, Xinzhi Zhang, Wang $\mathrm{Jia}$, and Tao Xu. Inkjet Bioprinting of Biomaterials. Chemical Reviews, 120(19):1079310833, October 2020. doi: 10.1021/acs.chemrev.0c00008.

163. Joshua W. Tashman, Daniel J. Shiwarski, and Adam W. Feinberg. A high performance open-source syringe extruder optimized for extrusion and retraction during FRESH 3D bioprinting. HardwareX, 9:e00170, April 2021. doi: 10.1016/j.ohx.2020.e00170.

164. Rania Taymour, David Kilian, Tilman Ahlfeld, Michael Gelinsky, and Anja Lode. 3D bioprinting of hepatocytes: core-shell structured co-cultures with fibroblasts for enhanced functionality. Scientific Reports, 11(1):5130, March 2021. doi: 10.1038/s41598-021-84384-6.

165. Jessica Nulty, Rossana Schipani, Ross Burdis, and Daniel J. Kelly. Bioinks and Their Applications in Tissue Engineering. In Declan M. Devine, editor, Polymer-Based Additive Manufacturing: Biomedical Applications, pages 187-218. Springer International Publishing, Cham, 2019. doi: 10.1007/978-3-030-24532-0_9. 

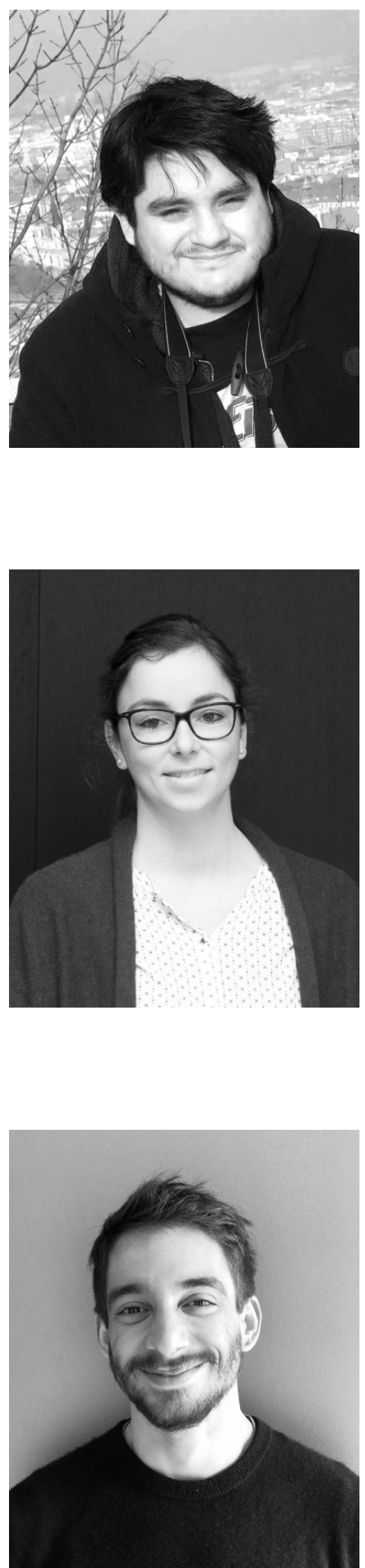

Mario Del Rosario graduated as a marine biologist from ESPOL (Ecuador) in 2012. He completed a MRes in Biomedical Sciences in the University of Glasgow (UK) in 2014 and stayed for a PhD in the Welcome Centre for Integrative Parasitology (UK) until 2019. During his PhD he worked in the invasion dynamics of Toxoplasma gondii combining parasite work, live imaging and super-resolution microscopy. He is currently a postdoctoral researcher in the Optical Cell Biology Lab at the Instituto Gulbenkian de Ciência (Portugal). He is interested in viral host-pathogen interactions and imaging approaches that reduces cell photodamage during image acquisition.

Hannah S. Heil received her M.Sc. in Nanotechnology from the Julius-Maximilian-University Würzburg (Germany) in 2015. She completed her Ph.D. in at the Graduate School of Life Science of the University of Würzburg in 2020 where she developed advanced imaging tools by combining biophotonics and super-resolution microscopy. She is currently a postdoctoral researcher in the Optical Cell Biology Lab at the Instituto Gulbenkian de Ciência (Portugal). Her current research interests include the development of automated and intelligent super-resolution microscopy to study host pathogen interactions during viral infection.

Afonso Mendes graduated in Biology at FCUL (2017, Portugal) and obtained a Master's degree in Biomedical Research at NOVA Medical School (2019, Portugal). He was selected for the IBB PhD Programme at IGC (2020, Portugal), where his passion for viruses combined with a growing desire to explore computational analysis and technology development led him to join Dr. Ricardo Henriques' laboratory as a PhD student. 

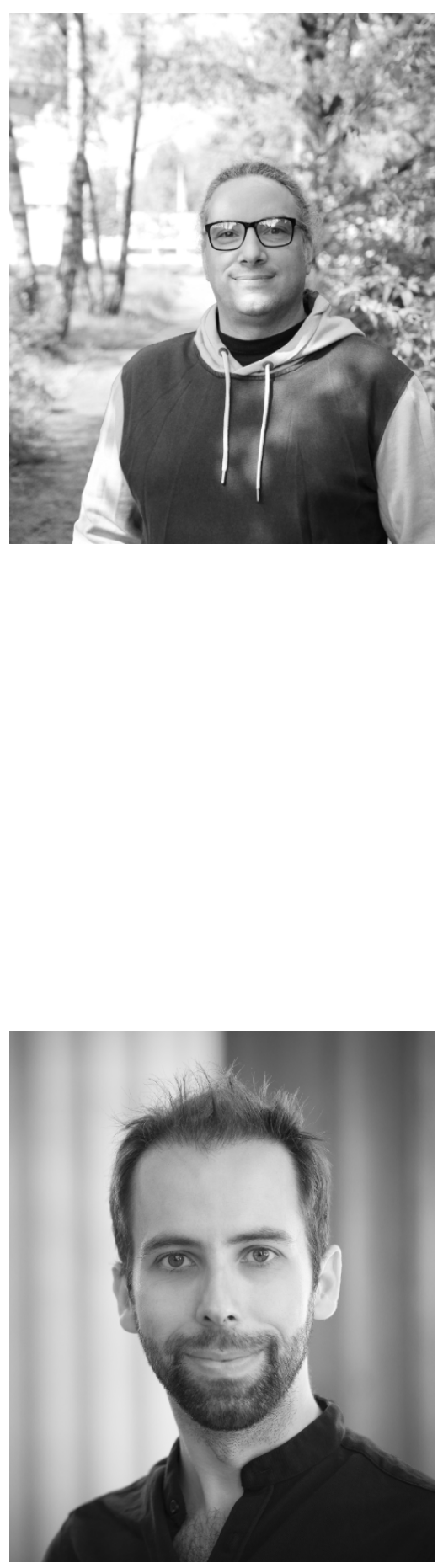

Dr. Vittorio Saggiomo obtained his MSc. degree in Organic Chemistry in 2007 at the University Federico II of Naples (Italy). He moved to Kiel (Germany) for pursuing his Ph.D. working on Dynamic Combinatorial Chemistry. In 2012, he started as postdoc at the university of Groningen (the Netherlands) in the field of Systems Chemistry. In 2013 he did a second postdoc at Wageningen University (the Netherlands). He is currently an Assistant Professor at the same University in the BioNanoTechnology group. His interests and expertises spread from organic chemistry to Open Technologies and 3D printing in microfabrication.

Prof. Ricardo Henriques graduated from Physics Engineering at FCUL (2006, Portugal). He carried out $\mathrm{PhD}$ research in optical physics and biophysics at IMM (2008, Portugal), CSIR (South Africa, 2008) and Institute Pasteur (France, 2008). He did a short postdoc in Institute Pasteur (2011, France), started a research group at UCL (2013, UK), and then a second laboratory at the Francis Crick Institute (2017, UK). Became full professor at UCL $(2018, \mathrm{UK})$ and recently started moving his laboratory to IGC (2020, Portugal) where he applies optical physics, machine-learning and cell biology to study viral infection. 